Published in final edited form as:

Nature. 2015 October 29; 526(7575): 710-714. doi:10.1038/nature15510.

\title{
CMT2D neuropathy is linked to the neomorphic binding activity of glycyl-tRNA synthetase
}

\author{
Weiwei He ${ }^{1,{ }^{*}}$, Ge Bai ${ }^{2,{ }^{*}}$, Huihao Zhou ${ }^{1}, \mathrm{Na} \mathrm{Wei}^{1}{ }^{1}$ Nicholas M. White ${ }^{2}$, Janelle Lauer ${ }^{3}$, \\ Huaqing Liu ${ }^{4}$, Yi Shi ${ }^{1}$, Calin Dan Dumitru ${ }^{1}$, Karen Lettieri ${ }^{2}$, Veronica Shubayev ${ }^{4}$, Albena \\ Jordanova $^{5}$, Velina Guergueltcheva ${ }^{6}$, Patrick R. Griffin ${ }^{3}$, Robert W. Burgess ${ }^{7}$, Samuel L. \\ Pfaff $^{2, \ddagger}$, and Xiang-Lei Yang ${ }^{1, \ddagger}$ \\ ${ }^{1}$ Departments of Chemical Physiology and Cell and Molecular Biology, The Scripps Research \\ Institute, La Jolla, CA 92037, USA \\ ${ }^{2}$ Howard Hughes Medical Institute and Gene Expression Laboratory, The Salk Institute for \\ Biological Studies, La Jolla, CA 92037, USA \\ ${ }^{3}$ Department of Molecular Therapeutics, The Scripps Research Institute, Jupiter, FL, 33458, USA \\ ${ }^{4}$ Department of Anesthesiology, University of California San Diego, La Jolla, CA 92093, USA \\ ${ }^{5}$ Molecular Neurogenomics Group, VIB Department of Molecular Genetics, University of Antwerp, \\ Antwerp, Belgium \\ ${ }^{6}$ Department of Neurology, Medical University of Sofia, Sofia, Bulgaria \\ ${ }^{7}$ The Jackson Laboratory, Bar Harbor, ME 04609, USA
}

\section{Summary}

\begin{abstract}
Selective neuronal loss is a hallmark of neurodegenerative diseases, which counter-intuitively are often caused by mutations in widely-expressed genes ${ }^{1}$. Charcot-Marie-Tooth (CMT) diseases are the most common hereditary peripheral neuropathies, for which there are no effective therapies ${ }^{2,3}$. A subtype of the diseases-CMT2D—is caused by dominant mutations in GARS, encoding the ubiquitously expressed enzyme glycyl-tRNA synthetase (GlyRS). Despite the broad requirement of GlyRS for protein biosynthesis in all cells, mutations in this gene cause a selective degeneration
\end{abstract}

\footnotetext{
Users may view, print, copy, and download text and data-mine the content in such documents, for the purposes of academic research, subject always to the full Conditions of use:http://www.nature.com/authors/editorial_policies/license.html\#terms

${ }^{\ddagger}$ Correspondence: Xiang-Lei Yang, The Scripps Research Institute, 10550 North Torrey Pines Road, La Jolla, CA 92037,

xlyang@scripps.edu, telephone: (858) 784-8976, Samuel L. Pfaff, The Salk Institute for Biological Studies, 10010 North Torrey Pines

Road, La Jolla CA 92037, pfaff@salk.edu, telephone: (858) 453-4100 x2018.

These authors contributed equally to this work

Supplementary Information is linked to the online version of the paper at www.nature.com/nature

Author Contributions

W.H., G.B., S.L.P. and X.-L.Y. designed the study, analyzed the data, and prepared the manuscript. W.H. carried out molecular cloning, binding analyses, secretion studies, and other biochemical experiments. G.B. performed the mouse genetics, viral injections, behavior testing, and histology experiments. H.Z. carried out Nrp1 domain mapping, GlyRS-VEGF competition, and additional pulldown assays, and contributed to study design and figure preparation. N. W. performed co-immunoprecipitation to detect aberrant GlyRS-Nrp1 interaction using CMT2D patient samples. A.J. and V.G. provided transformed lymphocytes samples from CMT2D patients. J.L. and P.G. performed the hydrogen-deuterium exchange analysis. N.M.W. and K.L. assisted with mouse studies. Y.S. and C.D.D. contributed to biochemical experiments. H.L. and V.S. contributed to histology experiments. R.W.B. provided mice, technical support, and scientific advice.
} 
of peripheral axons leading to deficits in distal motor function ${ }^{4}$. How mutations in GlyRS (GlyRS ${ }^{C M T 2 D}$ ) are linked to motor neuron vulnerability has remained elusive. Here we report that GlyRS CMT2D acquires a neomorphic binding activity that directly antagonizes an essential signaling pathway for motor neuron survival. We find that CMT2D mutations alter the conformation of GlyRS, enabling GlyRS ${ }^{\mathrm{CMT} 2 \mathrm{D}}$ to bind the Neuropilin 1 (Nrp1) receptor. This aberrant interaction competitively interferes with the binding of the cognate ligand vascular endothelial growth factor (VEGF) to Nrp1. Genetic reduction of Nrp1 in mice worsens CMT2D symptoms, whereas enhanced expression of VEGF improves motor function. These findings link the selective pathology of CMT2D to the neomorphic binding activity of GlyRS ${ }^{\text {CMT2D }}$ that antagonizes the VEGF/Nrp1 interaction, and indicate the VEGF/Nrp1 signaling axis is an actionable target for treating CMT2D.

CMT diseases are a group of inherited disorders that specifically affect the peripheral nervous system and are characterized by progressive weakness and atrophy in the hands and feet ${ }^{2,3}$. Recent progress in neurogenetic studies has uncovered aminoacyl-tRNA synthetases as the largest gene family implicated in CMT. Among them, GARS, encoding glycyl-tRNA synthetase (GlyRS) is the first member identified, whose mutations cause a dominant axonal form of CMT (CMT2D) $)^{4}$. The canonical function of this evolutionarily ancient cytoplasmic enzyme is to catalyze the ligation of glycine to the 3 '-end of its cognate tRNA as the first step of protein synthesis. Interestingly, emerging evidence has revealed that GlyRS in multicellular organisms, like several other tRNA synthease family members, has acquired the ability to be secreted from cells and as an extracellular protein can influence cell signaling ${ }^{5-9}$.

More than a dozen missense GARS mutations (GlyRS ${ }^{\mathrm{CMT} 2 \mathrm{D}}$ ) have been found in CMT2D patients with varying degrees of genetic evidence for disease-association ${ }^{10-13}$. Among them, three mutations (E71G, L129P and G240R) are the most tightly linked to the disease ${ }^{11}$ (Fig. 1a). Spontaneous and ENU-induced missense mutations in mouse Gars also cause CMT2Dlike phenotypes ${ }^{14,15}$ (Fig. 1a). These dominant mutations are found throughout the primary sequence of GlyRS, with some affecting the aminoacylation enzyme activity whereas others do not ${ }^{11}$. Mice with a heterozygous deletion of the Gars gene and a $50 \%$ reduction in glycyl-tRNA synthetase activity are normal ${ }^{15}$. Furthermore, overexpression of the wild-type GlyRS (GlyRS ${ }^{\text {WT }}$ ) in a CMT2D-disease mouse fails to rescue the neuropathy ${ }^{16}$. These genetic experiments indicate that CMT2D may arise from an abnormal activity gained by GlyRS $^{\text {CMT2D }}$ rather than a general defect in tRNA aminoacylation as initially suspected.

Like most class II tRNA synthetases, GlyRS functions as a dimer for aminoacylation. Interestingly, despite being dispersed in three separate domains of GlyRS, all known CMT2D-causing mutations are located near the dimer interface in the GlyRS crystal structure $^{17}$ (Fig. 1b, c). We found that 5 different human mutations associated with CMT2D caused a conformational opening in GlyRS that exposes new protein surfaces to solution ${ }^{17}$ (Fig. 1 b). To test if this conformational change also occurs in P234KY-GlyRS ${ }^{\text {CMT2D }}$ linked to CMT-like phenotypes in mice, we performed hydrogen-deuterium exchange labeling on P234KY-GlyRS and found that the mouse mutation likewise opens new surfaces of the 
GlyRS protein to solution (Fig. 1b, c, and Extended Data Fig. 1). These findings suggest that the abnormal "opening" is shared by many CMT2D mutants.

The new surfaces exposed by mutations in GlyRS ${ }^{\text {CMT2D }}$ could lead to neomorphic proteininteractions. This prompted us to search for binding partners unique to GlyRS ${ }^{\mathrm{CMT} 2 \mathrm{D}}$. We performed a candidate-protein screen by in vitro protein pull-down assays. Because motor neurons are the most frequently affected neuronal type in CMT2 $\mathrm{D}^{4,18,19}$, our initial screen focused on molecules that are highly expressed by motor neurons and that have been linked to motor neuron diseases/defects. We detected strong binding between the receptor Nrp1 and

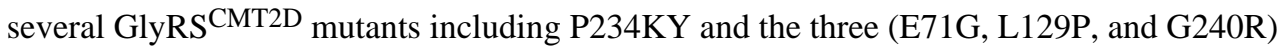
with the strongest link to CMT2D in patients ${ }^{11}$ (Fig. 2a and Extended Data Fig. 2a, b). In contrast, GlyRS $^{\mathrm{WT}}$ did not bind Nrp1 strongly, and GlyRS ${ }^{\mathrm{CMT} 2 \mathrm{D}}$ failed to bind to other motor neuron proteins such as TrkB, DCC, Robo1, and Unc5C (Fig. 2a and Extended Data Fig. 2a, b). To confirm that this binding-specificity occurs in vivo, we performed immunoprecipitations using neural tissues from WT and P234KY-CMT2D mouse littermates. Anti-Nrp1 antibodies co-precipitated significantly more GlyRS from CMT2D mice than WT controls (Fig. 2b), indicating P234KY-GlyRS CMT2D binds to Nrp1 in vivo. Furthermore, we also found that the GlyRS-Nrp1 interaction is significantly stronger in CMT2D patients carrying the L129P mutation than in healthy individuals (Fig. 2c). To quantify these interactions we used biolayer interferometry (BLI) and a biosensor with immobilized $\mathrm{Nrp} 1$ on the surface. GlyRS ${ }^{\mathrm{WT}}$ binding was undetectable at $1 \mu \mathrm{M}$; whereas GlyRS ${ }^{\text {CMT2D }}$ bound significantly stronger with a $K_{d}$ of $29.8 \pm 6.3 \mathrm{nM}$ for L129P and $208.7 \pm 53.8 \mathrm{nM}$ for P234KY.

Next, we mapped the site where GlyRS ${ }^{\mathrm{CMT} 2 \mathrm{D}}$ binds to Nrp1 using pull-down assays with domain-deletion constructs. Removal of the extracellular a and c domains of Nrp1 did not alter GlyRS ${ }^{\text {CMT2D }}$ binding, whereas the extracellular Nrp1-b1 domain alone was sufficient to bind P234KY-GlyRS CMT2D (Fig. 2d and Extended Data Fig. 2c). Because the b1 domain is the binding site of VEGF- $\mathrm{A}_{165}$, this finding raised the possibility that GlyRS ${ }^{\mathrm{CMT} 2 \mathrm{D}}$ might influence the binding of VEGF-A 165 to this region of Nrp1. Using pull-down assays, we found that increasing concentrations of P234KY or L129P GlyRS ${ }^{\mathrm{CMT} 2 \mathrm{D}}$ compete with VEGF-A $\mathrm{A}_{165}$ binding to the $\mathrm{b}$ domains of Nrp1 (Fig. 2e and Extended Data Fig. 2d). Conversely, increasing levels of VEGF-A 165 displaced P234KY or L129P-GlyRS CMT2D from the $b$ domains (Fig. 2 f and Extended Data Fig. 2d).

These observations led us to focus on GlyRS protein in the extracellular environment. Recent studies of GlyRS ${ }^{\mathrm{WT}}$ detected secretion from immune cells ${ }^{8}$, so we first examined whether GlyRS ${ }^{\mathrm{WT}}$ is released by cell types relevant to the peripheral nervous system and motor function. Indeed, endogenous GlyRS ${ }^{\mathrm{WT}}$ was detected in the culture media of mouse motor neuron and differentiated myotube cell lines, but not of undifferentiated myoblasts (Extended Data Fig. 3a-e). We found that secreted GlyRS ${ }^{\text {WT }}$ was enriched from extracellular sources using procedures that concentrate micro-vesicles $(30-100 \mathrm{~nm}$, "exosomes") (Extended Data Fig. 4a, b). Extracellular levels of GlyRSWT were diminished by application of the exosome-pathway inhibitor GW4869 and enhanced by exosomepathway activators monensin (Extended Data Fig. 3a-f). Next, we checked if CMT2Dcausing mutations affect the secretion of GlyRS ${ }^{\text {CMT2D }}$. Our results showed that P234KY- 
GlyRS $^{\text {CMT2D }}$ was detected at levels similar to GlyRS ${ }^{\mathrm{WT}}$ in the media of transfected cells (Extended Data Fig. 3g).

Nrp1 is a well-established receptor needed for motor neuron axon guidance and cell body migration $^{20,21}$. Our finding that GlyRS ${ }^{\mathrm{CMT} 2 \mathrm{D}}$ and VEGF-A 165 compete for access to Nrp1 raised the possibility that CMT2D-mice may phenocopy some features of VEGF- $\mathrm{A}_{164}$ (the mouse equivalent of human VEGF- $\mathrm{A}_{165}$ ) and Nrp1 mutant mice ${ }^{20}$. VEGF/Nrp1 signaling is necessary for the caudal migration of facial motor neurons from rhombomere (r) 4 to r6 during embryonic development (Fig. $2 \mathrm{~g}, \mathrm{j})^{20}$. This provided us with an excellent in vivo assay to examine the effect of GlyRS ${ }^{\mathrm{CMT} 2 \mathrm{D}}$ on a well-characterized system known to depend on VEGF/Nrp1 signaling. To track facial motor neuron migration we crossed CMT2D mice to transgenic ISL ${ }^{\text {MN }}$ :GFP-F reporter animals that selectively label facial motor neurons ${ }^{22,23}$. We found CMT2D mutant embryos developed at a normal rate, appeared overtly normal based on their overall morphology, and the expression levels of a variety of neuronal proteins were comparable to controls (Extended Data Fig. 5a, b). However, GFP-labeled facial motor neuron somata were found in ectopic anterior rhombomere locations in E13.5 GlyRS ${ }^{\mathrm{CMT} 2 \mathrm{D}}$ mutant embryos and manifested as an elongated stream across multiple rhombomeres (Fig. 2i). In contrast, most facial cells had completed their caudal migration to r6 in littermate controls at this stage (Fig. 2h). This observation was confirmed by immunostaining with the LIM homeodomain transcription factor Isl that is selectively expressed in the nuclei of facial branchiomotor neurons (Fig. 2k, 1 and Extended Data Fig. 5c). This facial motor neuron migration defect closely resembles the phenotypes of Nrp1-null and VEGF-A 164 -null mice as previously reported (Fig. $2 \mathrm{j})^{20}$. Taken together, the protein binding studies and embryological defects associated with

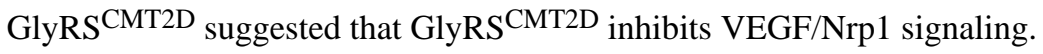

VEGF signaling is thought to protect neurons from a variety of damaging insults ${ }^{24}$. Intriguingly, deficient VEGF signaling leads to the selective degeneration of motor neurons in mice ${ }^{25}$. To examine whether the VEGF/Nrp1 pathway is involved in motor deficits that arise from GlyRS mutations, we tested whether a genetic interaction could be detected between Gars $^{C M T 2 D}$ and Nrp1. Although the data above suggest that GlyRS ${ }^{\text {CMT2D }}$ attenuates the normal Nrp1-signaling, it is still possible that GlyRS ${ }^{\mathrm{CMT} 2 \mathrm{D}}$ might activate aberrant signaling through Nrp1 and cause motor defects. In the first case motor phenotypes should get more severe as $\mathrm{Nrpl}$ gene dosage is reduced in CMT2D-mice, and in the second case motor phenotypes should improve. To test these possibilities, we intercrossed Gars ${ }^{C M T 2 D}$ mice with $\mathrm{Nrpl}$ heterozygous $\left(\mathrm{Nrpl}^{+/-}\right)$animals and characterized the motor behavior of the single and double mutant offspring. At 2 weeks when motor behavioral changes were not observed in either Gars $^{C M T 2 D}$ or $\mathrm{Nrp}^{+/-}$mutant mice, $20 \%$ of the compound heterozygous Gars ${ }^{C M T 2 D} / \mathrm{Nrpl}^{+/-}$mutant mice had developed neuromuscular dysfunction based on a hindlimb extension test (Extended Data Fig. 6a, b). At 4 weeks, CMT2D-like symptoms including overt neuromuscular dysfunction and an altered walking stride become apparent in $\operatorname{Gars}^{\mathrm{CMT} D}$ mutants, whereas $\mathrm{NrpI}^{+/-}$mice appeared normal (Fig. 3a-d and Supplementary Video 1, 2). Strikingly, by 4 weeks, 50\% of the Gars ${ }^{\text {CMT2D/ }}$ $\mathrm{Nrpl}^{+/-}$mutant mice had entirely lost the ability to spread their legs and toes (Fig. 3a and b), and exhibited severely abnormal gait patterns (Fig. 3c, d and Supplementary Video 3). 
After postnatal week 4, Gars ${ }^{C M T 2 D} / \mathrm{Nrpl}^{+/-}$mutant mice began to die. Consistent with our biochemical studies showing GlyRS ${ }^{\mathrm{CMT} 2 \mathrm{D}}$ binds poorly to other signaling receptors (see Fig. 2a and Extended Data Fig. 2a), intercrosses between $\operatorname{Fars}^{C M T 2 D}$ and $\operatorname{TrkB}^{+/-}, D C C^{+/-}$,

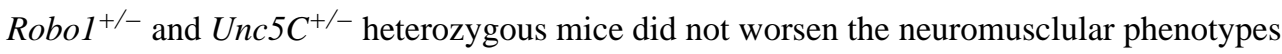
in the compound heterozygotes (Fig. 3b and Extended Data Fig. 6c).

The motor defects in Gars ${ }^{C M T 2 D}$ and Gars ${ }^{C M T 2 D} / \mathrm{Nrp1}^{+/-}$mutants were accompanied with significant pathological changes in the peripheral nerves and synaptic contacts with muscle fibers. Neuromuscular junctions (NMJs) displayed a normal apposition of nerve fibers and post-synaptic acetylcholine receptors in wild-type (WT) and $\mathrm{NrpI}^{+/-}$animals, while partially innervated and completely denervated NMJs were present in 4-week Gars ${ }^{C M T 2 D}$ mutants (Fig. 3e, f). The loss of nerve terminals at NMJs was markedly increased in Gars $^{C M T 2 D} / \mathrm{Nrpl}^{+/-}$mutants (Fig. 3e, f). Likewise, at 4 weeks of age many large-diameter axons were absent from the sciatic nerves of Gars $^{C M T 2 D}$ mutants compared to WT and $\mathrm{Nrpl}^{+/-}$littermates (Fig. 3g, h and Extended Data Fig. 7a-c). The absence of large diameter axons was even more dramatic in 4-week Gars ${ }^{C M T 2 D} / \mathrm{Nrpl}^{+/-}$compound mutants, and was comparable to the extreme axonal dystrophy observed in late-stage CMT2D mutants (Fig. $3 \mathrm{~g}$, h and Extended Data Fig. 7d) ${ }^{15}$. These findings demonstrate that $\mathrm{Nrpl}$ is an important genetic modifier of CMT2D pathology and that GlyRS ${ }^{\text {CMT2D }}$ antagonizes normal Nrp1signaling rather than activating aberrant signaling.

These findings prompted us to test whether VEGF overexpression could counteract GlyRS ${ }^{\text {CMT2D }}$ and help to slow the loss of motor function in CMT2D mice. A lentiviral vector encoding either VEGF-A 165 or GFP was injected bilaterally into the hindlimb muscles of Gars $^{C M T 2 D}$ mutant mice at postnatal day 5 prior to the onset of overt motor defects (Fig. 4a and Extended Data Fig. 8). By 4 weeks of age, we began to observe a reduction of limb strength in the control GFP-treated Gars ${ }^{C M T 2 D}$ animals using an inclined plane test (Fig. 4b). However, VEGF-A 165 -treated animals retained greater neuromuscular capacity with significantly higher scores (Fig. 4b). By 7 weeks, Gars ${ }^{C M T 2 D}$ animals exhibited a disrupted gait pattern with shortened hindlimb stride length, while VEGF- $\mathrm{A}_{165^{-}}$ treated animals maintained a significantly longer walking stride (Fig. 4c). Likewise, VEGF treatment significantly improved the motor performance of Gars ${ }^{C M T 2 D}$ mutants in the rotarod test (Fig. 4d). To minimize the possible influence from the natural variation in disease progression among individual animals, we compared the effect of VEGF treatment to GFP controls by separately treating each hindlimb from the same animal. Lentiviral vectors encoding VEGF-A 165 or GFP were injected unilaterally into each hindlimb of the same Gars ${ }^{C M T 2 D}$ mutant mouse at postnatal day 5 (Extended Data Fig. 9a). At 5 weeks, GFP-treated hindlimbs developed severe muscle weakness, largely losing their ability to extend. In contrast, the contralateral hindlimbs treated with VEGF-A 165 retained significant function (Extended Data Fig. 9b-e). These results suggest that VEGF treatment significantly ameliorates the loss of motor function in CMT2D mice.

A number of neurotropic factors have been tested as broad-spectrum strategies to enhance neuronal survival and treat motor diseases ${ }^{26-30}$. This raised the possibility that VEGF might slow the progression of CMT2D pathology by functioning as a generic trophic factor rather than a specific agent to restore normal VEGF/Nrp1-signaling. Therefore, we tested the 
effects of lentivirus-mediated expression of GDNF, a potent neurotrophin that has been used to enhance motor function and survival in mouse models of amyotrophic lateral sclerosis $^{26,27}$. Unlike VEGF-A 165 , we found GDNF failed to slow the disease progression in Gars $^{C M T 2 D}$ mice (Fig. 4b, d and Extended Data Fig. 9f). Next we tested whether VEGFmediated motor sparing is dependent upon Nrp1-binding by exploiting the binding specificity of VEGF protein isoforms. VEGF- $\mathrm{A}_{121}$ has overlapping functions with VEGF$\mathrm{A}_{165}$ but lacks high-affinity Nrp1 binding ${ }^{24}$. We found VEGF- $\mathrm{A}_{121}$ treatment failed to ameliorate the loss of motor function in CMT2D animals (Fig. 4b, d and Extended Data Fig. $9 \mathrm{~g})$. These data support a model in which VEGF treatment helps to guard against the motor loss arising from the aberrant activity of GlyRS ${ }^{\text {CMT2D }}$ by restoring VEGF/Nrp1 signaling.

Our results identify the $\mathrm{Nrpl}$ gene as an important genetic modifier for CMT2D, and link the selective pathology of this disease to the neomorphic binding of GlyRS ${ }^{\mathrm{CMT} 2 \mathrm{D}}$ to the receptor Nrp1. Although we found the same neomorphic binding to Nrp1 in several human GlyRS CMT2D mutants that are strongly disease-associative (Fig. 2a and Extended Data Fig. $2 \mathrm{a}, \mathrm{b}$ ), we cannot rule out the possibility that some GlyRS ${ }^{\mathrm{CMT} 2 \mathrm{D}}$ mutants may interact with other extracellular and/or intracellular targets. Nevertheless, our findings strongly suggest that the VEGF/Nrp1 pathway is an actionable target for treating CMT2D (Extended Data Fig. 10). While the exact role of VEGF in the motor system remains poorly defined, VEGFdeficient mice selectively develop symptoms of motor neuron disease over time ${ }^{25}$. The direct antagonism of VEGF/Nrp1 signaling by GlyRS ${ }^{\text {CMT2D }}$ found here further indicates that deficient VEGF-signaling may represent a common pathogenic pathway that is susceptible to abnormal activity in other motor neuron diseases. A broad implication from this work is that the molecular basis of selective neuronal vulnerability in neurodegenerative diseases may arise from the neomorphic activity of misfolded proteins interacting with susceptible signaling targets in specific cell types. This conceptual framework may be applied for identifying additional drugable-targets to treat neurodegenerative diseases including other forms of CMT.

\section{Methods}

\section{Mice}

The following strains of mice were used in this study: wild-type C57BL/6J (JAX), P234KYCMT2D mutant (background includes a mix of C57BL/6, CB6 and CAST) ${ }^{14}, \mathrm{Tg}$ $(\mathrm{Hb9} \text { :GFP) })^{31}, \mathrm{Tg}$ (ISL ${ }^{\mathrm{MN}}$ :GFP-F) ${ }^{23}$, Nrp1 mutant ${ }^{32}$, TrkB mutant ${ }^{33}$, Robo1 mutant ${ }^{34}$, DCC mutant ${ }^{35}$, and Unc5C mutant ${ }^{36}$. Both male and female mice were used in this study. All experiments were done in accordance with Institutional Animal Care and Use Committee animal protocols and BSL2+ safety protocols, on animals housed in groups on a 12-h lightdark cycle.

\section{Recombinant GlyRS expression and purification}

C-terminal His-tagged human GlyRS ${ }^{\mathrm{WT}}$ and GlyRS ${ }^{\mathrm{CMT} 2 \mathrm{D}}$ proteins were individually cloned into pET21b vector (Novagen) and expressed in Escherichia coli BL21 (DE3) host cells at $25^{\circ} \mathrm{C}$. The proteins were purified by Ni-NTA agarose affinity column followed by ion exchange monoQ column and size exclusion column Superdex 200 (GE Healthcare). To 
prepare non-tagged human GlyRS ${ }^{\mathrm{WT}}$ and GlyRS ${ }^{\mathrm{CMT} 2 \mathrm{D}}$ proteins, the GlyRS gene was fused with a N-terminal His-SUMO tag, cloned into pET28a vector (Novagen), and expressed as His-SUMO-GlyRS fusion proteins in Escherichia coli BL21 (DE3) cells. The fusion proteins were purified with a Ni-NTA agarose affinity column, and then subjected to homemade Ulp1 protease to remove the His-SUMO tag. The non-tagged GlyRS proteins were separated from the tag by flowing through the Ni-NTA column again.

\section{Hydrogen-Deuterium Exchange (HDX) analysis}

Solution-phase amide HDX was performed with a fully automated system as described previously ${ }^{37}$. Briefly, $4 \mu \mathrm{L}$ of His-tagged GlyRS ${ }^{\mathrm{P} 234 \mathrm{KY}}$ or GlyRS ${ }^{\mathrm{WT}}$ was diluted to $20 \mu \mathrm{L}$ with $\mathrm{D}_{2} \mathrm{O}$-containing $\mathrm{HDX}$ buffer to a final concentration of $10 \mu \mathrm{M}$, and incubated at $4{ }^{\circ} \mathrm{C}$ for 10,30,60,900, and 3,600 seconds. Following on-exchange, unwanted back exchange was minimized by adding $30 \mu \mathrm{L}$ of $1 \%$ TFA in $5 \mathrm{M}$ urea to denature the protein (held at 1 $\left.{ }^{\circ} \mathrm{C}\right)$. Samples were then passed across an immobilized pepsin column (prepared in house) at $50 \mu \mathrm{L} \mathrm{min}{ }^{-1}\left(0.1 \% \mathrm{TFA}, 15^{\circ} \mathrm{C}\right)$, and the resulting peptides were trapped onto a $\mathrm{C} 8$ trap cartridge (Thermo Fisher, Hypersil Gold). Peptides were eluted across a $1 \mathrm{~mm} \times 50 \mathrm{~mm}$ C18 HPLC column (Hypersil Gold, Thermo Fisher) with a $4-40 \% \mathrm{CH}_{3} \mathrm{CN}$ gradient and $0.3 \%$ formic acid over $5 \mathrm{~min}$ at $2^{\circ} \mathrm{C}$, and electrosprayed directly into an Orbitrap mass spectrometer (LTQ Orbitrap with ETD, Thermo Fisher). Data were processed with in-house software $^{38}$ and visualized with PyMOL (DeLano Scientific). The difference in HDX between GlyRS ${ }^{\mathrm{P} 234 \mathrm{KY}}$ and GlyRS ${ }^{\mathrm{WT}}$ was calculated by subtracting the average percentage deuterium uptake for GlyRS ${ }^{\mathrm{P} 234 \mathrm{KY}}$ from that for GlyRS ${ }^{\mathrm{WT}}$ following 10, 30, 60, 300, 900 and 3,600 seconds of on-exchange. Please note that we cannot directly compare the numbers obtained here for GlyRS ${ }^{\mathrm{P} 234 \mathrm{KY}}$ with those for other GlyRS ${ }^{\mathrm{CMT} 2 \mathrm{D}}$ mutants from the previous study ${ }^{17}$, because this and the precious analysis were carried out in two different laboratories with different instruments and experimental procedures.

\section{Detection of GlyRS proteins in cell cultures}

NSC-34 motor neuron cells (Cellutions Biosystems Inc.) and C2C12 mouse adherent myoblasts (From Dr. Ardem Patapoutian's lab at The Scripps Research Institute) were maintained in Dulbecco's modified Eagle's medium (DMEM) supplemented with $10 \%$ heatinactivated fetal bovine serum (FBS) and $1 \%$ penicillin (Life Technologies) at $37^{\circ} \mathrm{C}$ in humidified incubator containing $5 \% \mathrm{CO}_{2}$. These cell lines had not been recently authenticated and tested for mycoplasma contamination. Myogenic differentiation of $\mathrm{C} 2 \mathrm{C} 12$ myoblasts was induced by substituting the FBS with $2 \%$ horse serum. The cells were further cultured in Opti-MEM Reduced Serum Medium (Life Technologies) for $16 \mathrm{~h}$. Brefeldin A (Cell Signaling Technology), GW4869 (Sigma-Aldrich), or monensin (eBioscience) was added to the cell medium for $2 \mathrm{~h}$ before the cells and the medium were separated for Western-blot analysis. After removal of cell debris by spinning the medium at $300 \times \mathrm{g}$ for 10 min, the supernatant was concentrated using Amicon Ultra-4 Centrifugal filter (Millipore). Cells were lysed using cell lysis buffer (ATCC) with added protease inhibitor cocktail (Roche). The following antibodies were used for Western-blot analysis: mouse anti-GlyRS (H00002617-B01P, ABNOVA; 1:1000), Rabbit anti-GAPDH (\#3683, Cell Signaling Technology; 1:1000), Rabbit anti-vWF (sc-14014, Santa Cruz; 1:50), and Rabbit antiTSG101 (MABC649, Millipore; 1:1000). To study the effect of CMT2D-causing mutation 
on GlyRS secretion, constructs overexpressing V5-tagged GlyRS ${ }^{\mathrm{P} 234 \mathrm{KY}}$ or GlyRS ${ }^{\mathrm{WT}}$ were transfected into COS7 cells using lipofectamine 2000 (Invitrogen). The expression and secretion of GlyRS proteins were detected by Western blot analysis using anti-V5 antibody (R960-25, Invitrogen; 1:5000).

\section{Exosome purification and analysis}

The general idea of exosome purification by differential centrifugation is depicted in Extended Data Fig. 3a. Supernatants from NSC-34 cell media were subjected to successive centrifugation steps at $4{ }^{\circ} \mathrm{C}$ : 1) $200 \times \mathrm{g}$ for $10 \mathrm{~min}$ to eliminate floating cells; 2) $2,000 \times \mathrm{g}$ for $10 \mathrm{~min}$ to discard large dead cells; 3) $10,000 \times \mathrm{g}$ for $1 \mathrm{~h}$ to remove cell debris and cellular organelles such as mitochondria and lysosomes. At each step, the pellet was thrown away and the supernatant was used for the following step. The final supernatant was centrifuged at $100,000 \times \mathrm{g}$ at $4{ }^{\circ} \mathrm{C}$ for $4 \mathrm{~h}$ to pellet micro-vesicles that are commonly known as "exosomes". The final supernatant and the exosome fraction were analyzed by Western blot analysis using antibodies specified above and Rabbit anti-Bip antibody (\#3183S, Cell signaling technology; 1:1000).

\section{In vitro pull-down assay}

Recombinant rat Nrp1-Fc, mouse TrkB-Fc, mouse DCC-Fc, rat Robo1-Fc and human Unc5c-Fc extracellular domain-Fc chimeras (R\&D systems) were bound to the Protein G beads. Purified non-tagged GlyRS ${ }^{\mathrm{WT}}$ and GlyRS ${ }^{\mathrm{CMT} 2 \mathrm{D}}$ proteins were individually added to the receptor-immobilized beads and incubated for $1 \mathrm{~h}$ at $4^{\circ} \mathrm{C}$. After removal of unbound GlyRS proteins, SDS-loading buffer was directly added to the beads to elute the receptor and its bound GlyRS. The amount of GlyRS bound to receptors was analyzed by Westernblot analysis using mouse anti-GlyRS antibodies (H00002617-B01P, ABNOVA; 1:1000).

\section{Co-immunoprecipitation and western-blot analysis of protein expression in mouse tissues}

The interaction between endogenous GlyRS and Nrp1 proteins was detected by coimmunoprecipitation. Adult mouse neural samples were lysed using RIPA buffer (Cell Signaling Technology) containing $20 \mathrm{mM}$ Tris- $\mathrm{HCl}$ (pH 7.5), $150 \mathrm{mM} \mathrm{NaCl}, 1 \mathrm{mM}$ $\mathrm{Na}_{2}$ EDTA, 1 mM EGTA, $1 \%$ NP-40, $1 \%$ sodium deoxycholate, $2.5 \mathrm{mM}$ sodium pyrophosphate, $1 \mathrm{mM} \beta$-glycerophosphate, $1 \mathrm{mM} \mathrm{Na}_{3} \mathrm{VO}_{4}$, and $1 \mu \mathrm{g} / \mathrm{mL}$ leupeptin. Immunoprecipitation was performed with rabbit anti-Nrp1 antibody (NBP1 40666, Novus Biologicals; $1: 100$ ) and the precipitates were subjected to Western-blot analysis using mouse anti-GlyRS antibody (H00002617-B01P, ABNOVA; 1:1000).

Western-blot was performed to analyze the expression levels of various neuronal proteins in E12.5 WT and CMT2D mutant neural tissues. The following primary antibodies were used: mouse anti-GlyRS (H00002617-B01P, ABNOVA; 1:1000), rabbit anti-Nrp1 (\#3725S, Cell Signaling; 1:1000), rabbit anti-VEGFR1 (\#36-1100, Life Technologies; 1:1000), rabbit antiVEGFR2 (\#2479S, Cell Signaling; 1:1000), mouse anti- $\beta$-actin (\#3700, Cell Signaling; 1:1000), mouse anti-DCC (AF5, Abcam; 1:100), rabbit anti-Robo1 (NB100-60458, Novus Biologicals; 1:2000), mouse anti-NF (2H3, DSHB; 1:100), mouse anti-MAP2 (MAB364, Millipore; 1:500), rabbit anti-GAP43 (AB5220, Millipore; 1:1000), and rabbit anti- $\beta$-catenin (\#9587, Cell signaling technology; 1:1000). 


\section{Co-immunoprecipitation using CMT2D patient samples}

Peripheral blood was drawn from CMT2D patients carrying the L129P mutation and control individuals after obtaining their written informed consent. The study complies with the ethical guidelines of the Medical University of Sofia, Bulgaria and University of Antwerp, Belgium. Lymphocytes were isolated on a Ficoll-Paque gradient, transformed with EpsteinBarr virus and incubated at $37^{\circ} \mathrm{C}$ for $2 \mathrm{~h}$. After centrifugation, cells were re-suspended in 4 $\mathrm{ml}$ RPMI complete medium (Life Technologies) supplemented with $1 \%$ phytohaemagglutinin. Cells were seeded on a 24 -well plate and incubated at $37^{\circ} \mathrm{C}, 6 \% \mathrm{CO}_{2}$ for a minimum of 3 days. After establishment, cell lines were cultivated in RPMI1640 medium containing $15 \%$ fetal calf serum, $1 \%$ sodium pyruvate, $1 \% 200 \mathrm{M} \mathrm{L}$-glutamine and $2 \%$ penicillin/streptomycin. The harvested lymphoblastoid cells were lysed using RIPA buffer (Cell Signaling Technology). Immunoprecipitation was performed with rabbit antiNrp1 antibody (Novus Biologicals) and rabbit anti-IgG (\#2729, Cell Signaling Technology) and the pull-downed samples were subjected to Western-blot analysis using rabbit antiGlyRS antibody (sc-98614, Santa Cruz Biotechnology, 1:500).

\section{Mapping of GlyRS ${ }^{\text {CMT2D }}$ interaction domain on Nrp1}

The variants of Nrp1 extracellular domain (ECD) include intact ECD (res. Arg23-Asp840), b1b2c domain (res. Phe273-Asp840), a1a2 domain (res. Arg23-Asp272), b1b2 domain (res. Phe273-Phe643), c domain (res. Thr589-Asp840), b1 domain (res. Phe273-Asp428) and b2 domain (res. Lys425-Phe643). These variants were designed as chimera proteins containing a 17-residue secretion signal peptide from myeloid cell surface antigen CD33 (gp67) at the $\mathrm{N}$-terminus and a human $\mathrm{IgG} \mathrm{Fc}$ domain at $\mathrm{C}$-terminus, and were expressed using pcDNA6.0/V5-His-A vector (Life Technologies). For each Nrp1 variants, $3 \mu \mathrm{g}$ plasmids were transfected using Lipofactmine 2000 (Life Technologies) into human HEK293 cells in a 6-well plate. $20 \mathrm{~h}$ after transfection, MEM media containing secreted Nrp1 variants were collected and incubated with $30 \mu \mathrm{L}$ Protein A resins. The Nrp1-bound resins were divided equally into two $1.5 \mathrm{~mL}$ eppendorf tubes and incubated with $5 \mu \mathrm{g}$ of recombinant GlyRS $^{\text {CMT2D }}$ or GlyRS ${ }^{\mathrm{WT}}$ in $1 \mathrm{~mL}$ of Washing Buffer (PBS, $5 \mathrm{mM} \beta$-ME, $0.2 \%$ BSA and $0.05 \%$ Triton X-100) for $1 \mathrm{~h}$. Resins were then washed three times with the Washing Buffer and one time with PBS. The bound proteins were eluted with $30 \mu \mathrm{L}$ of SDS-PAGE sample buffer and subjected to Western-blotting analysis using mouse anti-GlyRS (H00002617B01P, ABNOVA; 1:1000) and Rabbit anti-His antibodies (RHIS-45P-Z, ICL Lab; 1:10000) to detect GlyRS and the Nrp1 variants, respectively.

The b1 (res. Phe273-Asp428), b2 (res. Lys425-Glu586), and b1b2 domain (res. Phe273Glu586) of Nrp1 fused with an N-terminal GST tag was cloned into the pET28a vector (Novagen), expressed in E. coli BL21(DE3) cells and purified with GST resin (Qiagen). GST or GST-Nrp1 fusion proteins was incubated with $20 \mu \mathrm{L}$ GST resin and then bind with non-tagged WT or P234KY GlyRS in $1 \mathrm{~mL}$ of Washing buffer (1 X PBS, $5 \mathrm{mM}$ BME, $0.2 \%$ BSA and $0.05 \%$ Triton X-100) for $1 \mathrm{~h}$. GST resins were washed three times with Washing buffer and one more time with PBS. The bound proteins were eluted with SDS-PAGE sample buffer, and subjected to West blotting analysis. 


\section{Competition assay between VEGF-A 165 and GlyRS ${ }^{\text {CMT2D }}$ for Nrp1 binding}

In each experiment, $5 \mu \mathrm{g}$ of GST-b1b2 protein was bound with $15 \mu \mathrm{L}$ of GST resin in $1 \mathrm{~mL}$ Washing Buffer on ice. The competition was tested in both directions. In one direction, 30 $\mathrm{nM}$ of P234KY GlyRS ${ }^{\mathrm{CMT} 2 \mathrm{D}}$ was added to GST-b1b2 with an increasing concentration of human VEGF-A 165 (IBL); in the opposite direction, $30 \mathrm{nM}$ of VEGF-A 165 was added with an increasing concentration of P234KY GlyRS ${ }^{\mathrm{CMT} 2 \mathrm{D}}$. After the resins were washed three times with the Washing buffer and one time with PBS, proteins were eluted with SDSPAGE sample buffer, and analyzed by Western-blot using rabbit anti-VEGF-A (ABS82, Millipore; 1:2000), mouse anti-GlyRS (H00002617-B01P, ABNOVA; 1:1000) and rabbit anti-GST (\#2622, Cell Signaling Technology; 1:1000) antibodies.

\section{Immunostaining and imaging}

Immunostaining of NMJs was performed as described ${ }^{14}$. Cocktails of the following primary antibodies were used to visualize nerves: rabbit anti-NF (AB1991, Millipore; 1:1000), rabbit anti-Synaptophysin (A0010, Dako; 1:2000), and mouse anti-SV2 (DSHB, 1:1000). Secondary antibodies were Alexa-488 or -647 conjugated (Molecular Probes/Invitrogen; 1:1000). Tetramethylrhodamine-conjugated a-bungarotoxin (T-1175, Molecular Probes/ Invitrogen; 1:1000) was used to visualize acetylcholine receptors (AChRs) on muscles. The occupancy of NMJs is measured by examining the overlap of the motor nerve terminal (green) with AChRs on the muscle (red). At least 40 randomly selected NMJs were examined from each of three mutant and three control mice. The flat-mount preparations of hindbrains were performed as previously described ${ }^{23}$. Rabbit anti-Is $11 / 2^{39}$ was used to label facial motor nuclei by whole-mount immunostaining. The distance between facial motor nucleas and trigeminal nucleas was measured for each embryo. Each distance was further normalized to relative distance of WT facial motor nucleas. Rabbit anti-VEGF (ab52917, Abcam, 1:200) was used to determine the expression of VEGF in muscle fibers.

Bright field and fluorescence images of whole embryos were obtained using a $0.8 \times$ objective on Zeiss Lumar.V12 fluorescence stereomicroscope. Confocal images were obtained using 10x and 20x objectives on Olympus Fluoview 1000 confocal microscope.

\section{Hindlimb extension test}

Mice were suspended by the tail and the extent of hindlimb extension was observed over 10 s. A score of 2 corresponded to a normal extension reflex in hindlimbs with splaying of toes. A score of 1 corresponded to clenching of hindlimbs to the body with partial splaying of toes. A score of 0 corresponded to clasping hindlimbs with curled toes. Three tests were performed for each mouse with 5-s intervals. A score of 1.5 or 0.5 corresponded to behaviors between 2 and 1 , or between 1 and 0 , respectively.

\section{Footprint test}

Blue ink was applied to the hind paws of each mouse and the animal was placed in a narrow alley $(9 \times 80 \times 25 \mathrm{~cm})$ with the floor covered with white paper. A home cage was placed at the end of the alley for the animal to walk to while leaving its footprints on the paper. Stride length was assessed by measuring the average distances of at least three consecutive steps on each side. 


\section{Inclined plane test}

Hindlimb strength was assessed at postnatal 4 weeks using the inclined plane test. Briefly, animals were placed on an inclined plane, and the angle of incline was gradually increased starting from $15^{\circ}$. The maximum angle at which the animal could maintain its position for 5 sec constituted the inclined plane score. The test was performed 3 times for each mouse.

\section{Rotarod test}

Motor coordination was assessed with a rotarod apparatus (Economex, Columbus Instruments). The mice were first placed on the stationary rod $(0 \mathrm{rpm})$ to acclimate them to the apparatus, followed by a trial at a rotation speed of $1 \mathrm{rpm}$ for $3 \mathrm{~min}$ or until a fall occurred. For testing, the rotation of the rotarod was accelerated from $0 \mathrm{rpm}$ with an accelerating rate $(0.1 \mathrm{rpm} / \mathrm{min})$. Latency of each mouse to fall was monitored for three consecutive trials and the intra-trial interval for each animal was about $20 \mathrm{~min}$. The average time of three trials was used as a measure of motor performance.

\section{Virus preparation and injection}

The cDNAs encoding GDNF, VEGF-A ${ }_{121}$, or VEGF-A 165 were cloned into lentiviral vector (p156RRLsinPPTCMVGFPPRE) between BamHI and SalI sites. All lentiviruses were produced by GT3 core facility at Salk Institute with a titer of $1 \times 10^{12}-2 \times 10^{13}$ genome copies/mL. Injections were performed at P5 ( \pm 1 day) after anesthetizing pups on ice. Multiple injections ( $\mathrm{n} \geq 8)$ of virus $(5 \mu \mathrm{L}$ for each limb) into a variety of hindlimb muscles were performed with a Hamilton syringe. Based on the expression pattern of GFP reporter, we found that the lentiviruses mainly infect muscle fibers.

\section{Nerve Histology and Imaging}

Mouse sciatic nerves were dissected and fixed in $2.5 \%$ glutaraldehyde in $0.1 \mathrm{M}$ phosphate buffer. Nerve samples were then osmicated, dehydrated, and embedded in araldite resin. Transverse nerve sections $(1 \mu \mathrm{m})$ were cut on a Leica RM2065 microtome and stained with methylene blue Azure II. Images were collected on a Leica DMR microscope or an Olympus BX61 microscope. Axon numbers were determined from two non-overlapping fields $(50 \times 50$ $\mu \mathrm{m})$ from each of three mutant and three control nerve samples. Axon diameters were measured by Image $\mathrm{J}$.

\section{Statistics}

All graphs and data generated in this study were analyzed using GraphPad Prism 6.0 Software (MacKiev) or Excel (Microsoft). Two-tailed unpaired t-tests with Welch's correction using parametric distribution, two-tailed Mann-Whitney test using unparametric distribution, or two-tailed paired Wilcoxon test using unparametric distribution were performed to measure differences from at least three independent biological replicates. P < 0.05 was considered significant. These tests do not require similar variance of the data between the groups that are being statistically compared. The normality of the data was determined by D'Agostino-Pearson omnibus test and Kolmogorov-Smirnov test. No statistical methods were used to predetermine sample sizes, but our sample sizes are similar to those generally used in the field. 
For all animal studies, analyses were performed on approximately equal numbers of male and female mice selected randomly from populations, and no sex-specific differences in the disease progression were identified. All behavioral experiments were performed in doubleblind fashion, and stressed animals were excluded from the analysis.

\section{Extended Data}

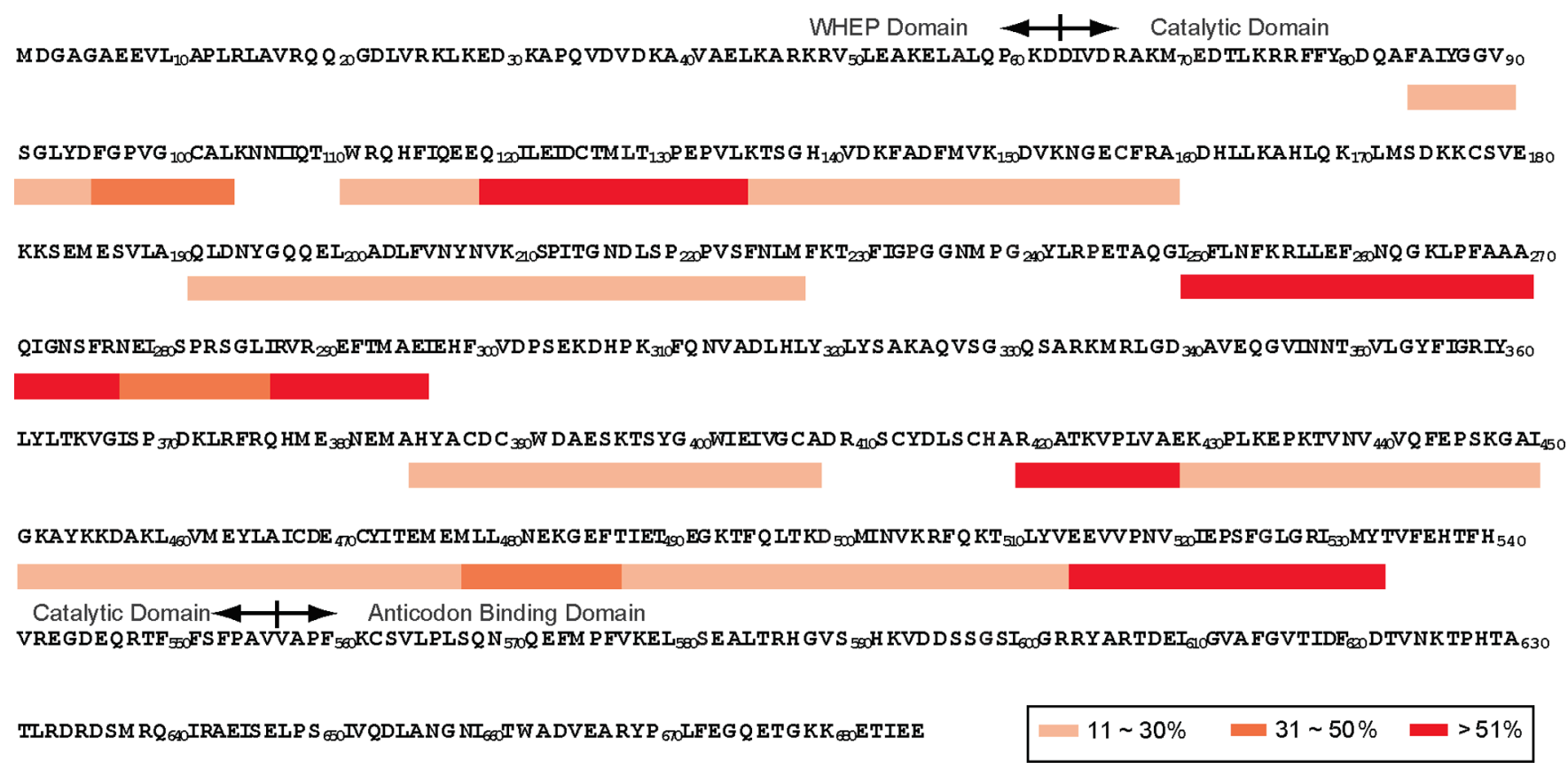

Extended Data Figure 1. Hydrogen-deuterium exchange analysis to compare P234KYGlyRS CMT2D and GlyRS $^{\text {WT }}$ in solution

A global increase (15\%) in deuterium incorporation for the mutant GlyRS was observed indicating overall structural opening. The regions having significant changes $(>10 \%)$ in deuterium incorporation are highlighted under the human cytosolic GlyRS sequence with different color codes (see box). 
a

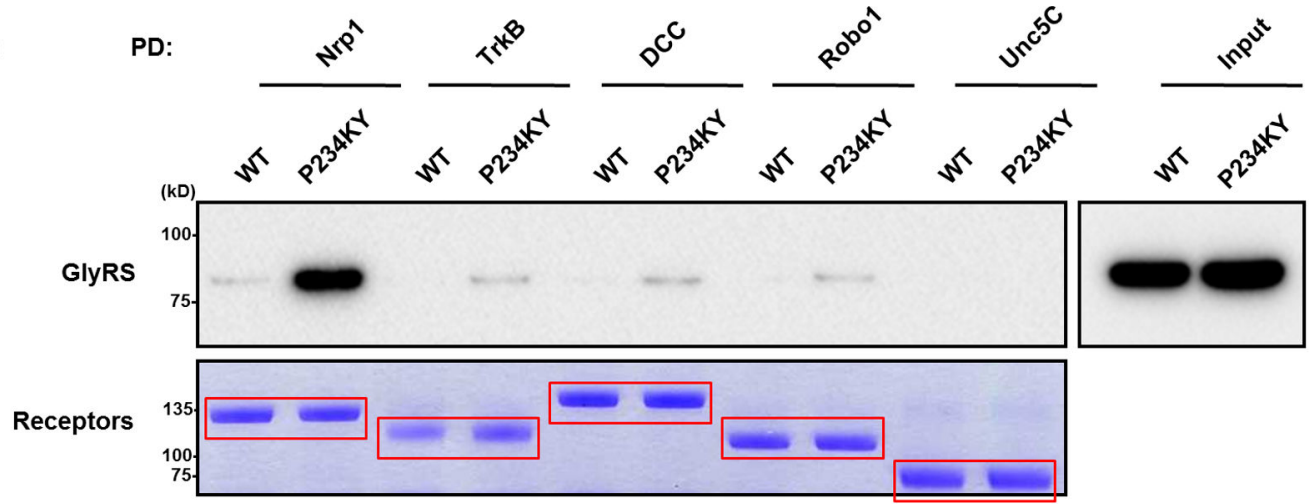

b d
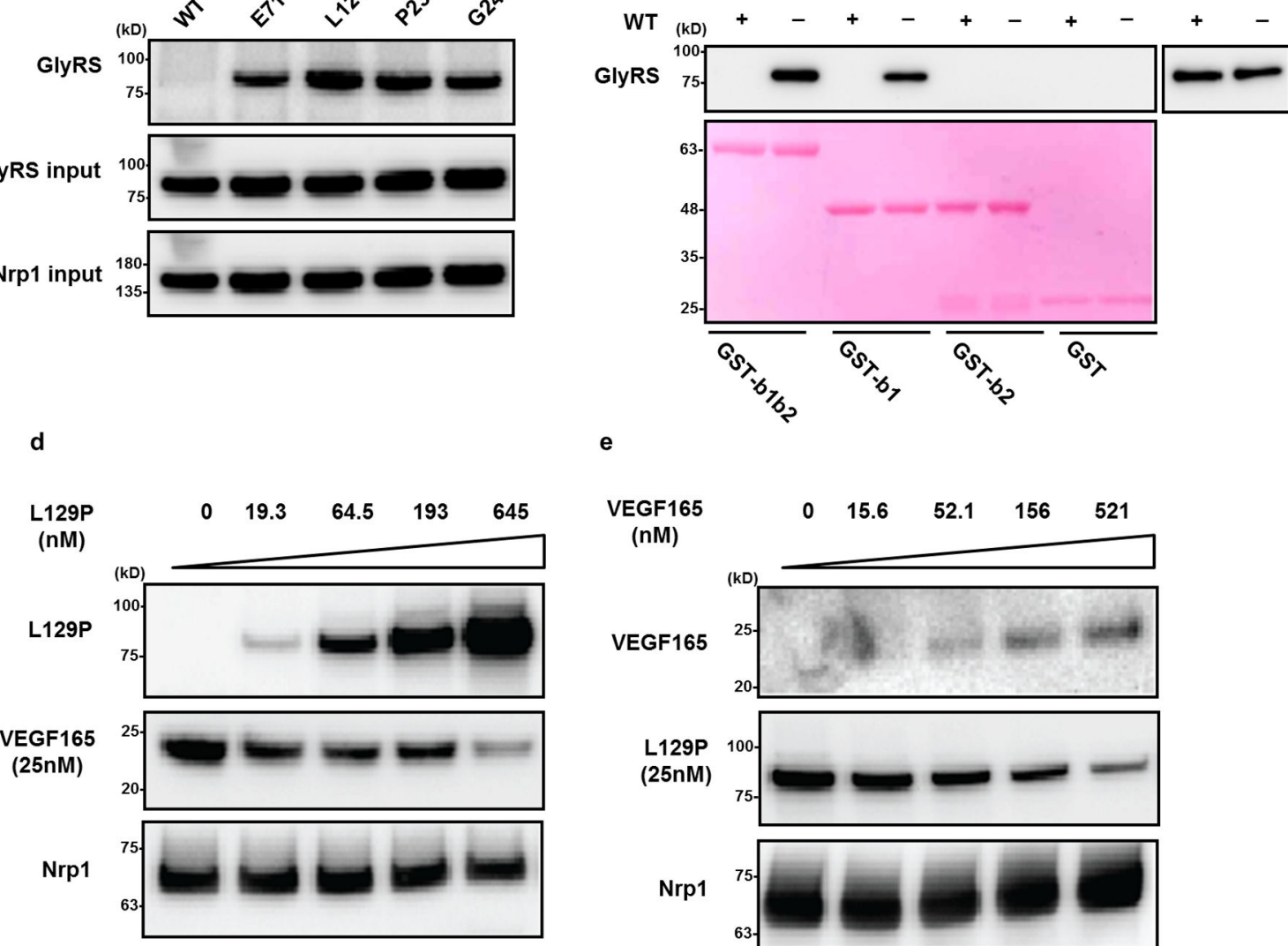

GlyRS input

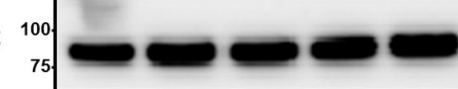

Nrp1 input
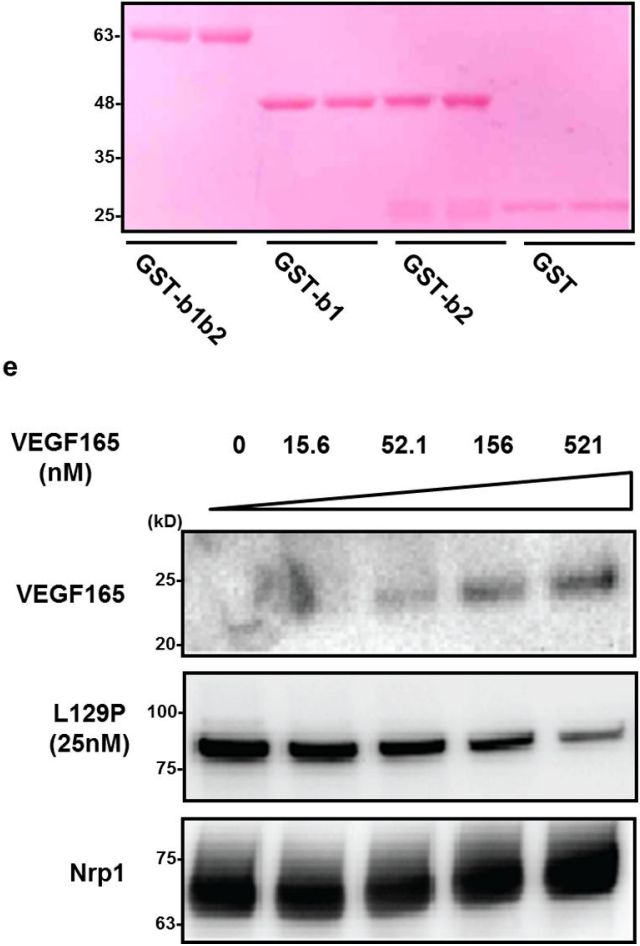

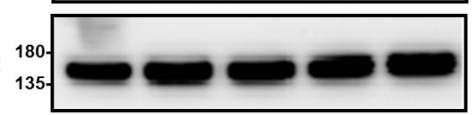

Extended Data Figure 2. Characterization of the binding activity of GlyRS CMT2D

a, In vitro pull-down of $\mathrm{P} 234 \mathrm{KY}-G l y R S^{\mathrm{CMT} 2 \mathrm{D}}$ proteins with the ectodomains of $\mathrm{Nrp1}$, TrkB, DCC, Robo1 and Unc5C proteins. Note the much stronger binding of GlyRS CMT2D with Nrp1 compared to other receptors. GlyRS was detected by immunoblot with antiGlyRS antibody; similar amounts of input receptors were visualized by Coomassie Brilliant Blue staining. $\mathbf{b}$, In vitro pull-down of GlyRS ${ }^{\mathrm{CMT} 2 \mathrm{D}}$ proteins with the ectodomain of Nrp1. In addition to L129P and P234KY, direct binding to Nrp1 was detected for E71G and G240R GlyRS ${ }^{\text {CMT2D }}$. c, GST pull-down to confirm that b1 domain of Nrp1 is the main 
binding site of GlyRS ${ }^{\text {CMT2D }}$. The amount of GST and GST fusion proteins used for GlyRS ${ }^{\text {CMT2D }}$ binding was visualized by Ponceau staining. d,e, In vitro pull-down assay showing the mutual competition between L129P-GlyRS ${ }^{\text {CMT2D }}$ and VEGF-A 165 for Nrp1 binding.

a

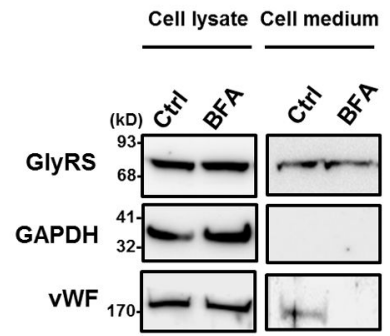

c

C2C12 myotubes

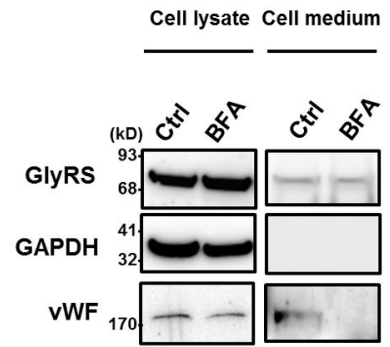

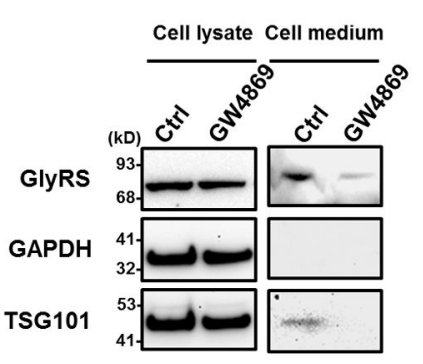

GlyRS

GAPDH

TSG101

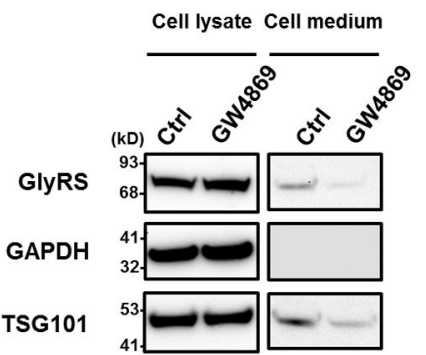

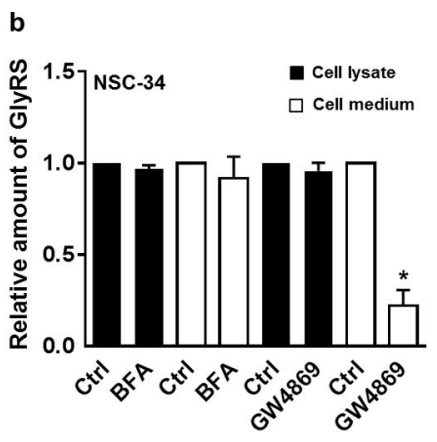

d

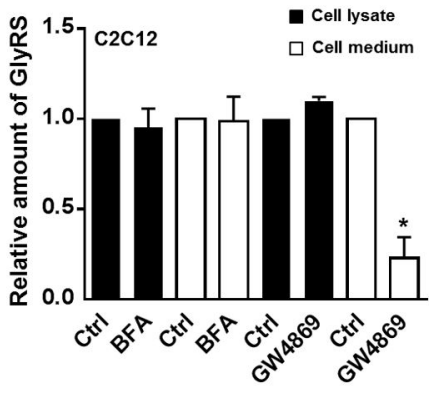

e

C2C12 myoblasts

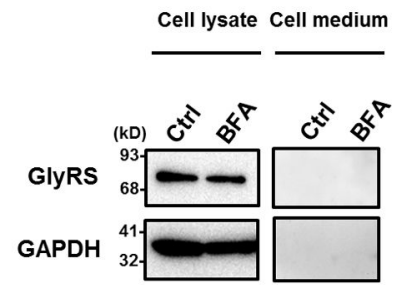

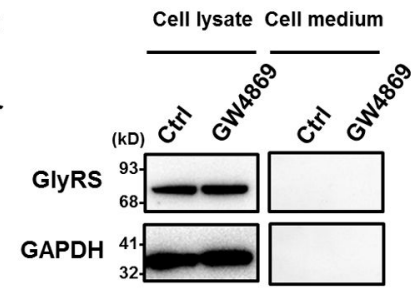

f NSC-34 motor neurons

g $\quad$ Cos-7
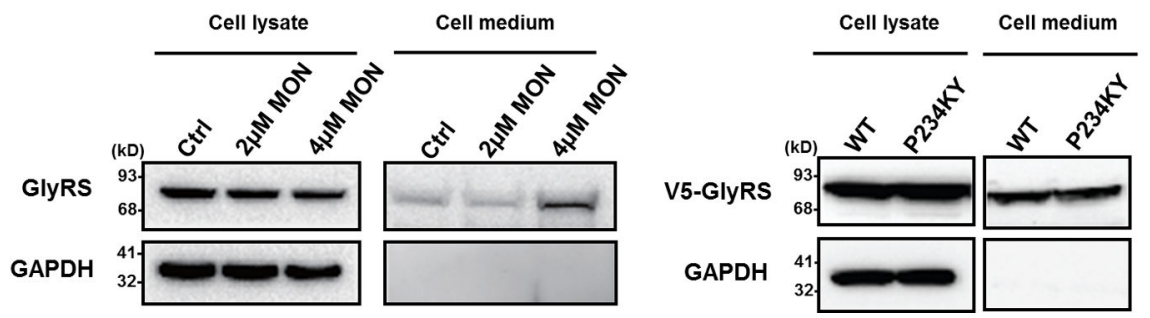

Extended Data Figure 3. Detection of GlyRS proteins in the cell medium

a, c, e, Western-blot analysis of the GlyRS protein levels in NSC34 motor neurons (a),

$\mathrm{C} 2 \mathrm{C} 12$ cell-differentiated myotubes $(\mathbf{c})$ and undifferentiated $\mathrm{C} 2 \mathrm{C} 12$ myoblasts $(\mathbf{e})$. The level 
of GlyRS proteins in cell medium is diminished by application of the exosome-pathway inhibitor GW4869, but not by Brefeldin A (BFA), an inhibitor of the classical endoplasmic reticulum (ER) to Golgi secretory pathway. GAPDH (cytoplasmic protein), vWF (secretory protein through ER-Golgi pathway) and TSG101 (Exosomal protein) are used as controls. b, d, Quantification of GlyRS protein level indicated in a c. Data are presented as the mean \pm SEM of three independent experiments ( ${ }^{*} \mathrm{p}<0.05$, t-test). f, Western-blot analysis of the GlyRS protein level in NSC34 motor neurons. The level of GlyRS proteins in the cell medium is increased by the treatment of monensin (MON), an activator for microvesicle release by regulating the intracellular calcium level ${ }^{40,41}$. Vehicle-treated cells were used as control (Ctrl). g, Western-blot analysis of the GlyRS protein level in Cos7 cells transfected with plasmids encoding GlyRS ${ }^{\mathrm{WT}}$ and P234KY-GlyRS ${ }^{\mathrm{CMT} 2 \mathrm{D}}$. The expression of GlyRS proteins was detected by immuno-blot with antibody to V5 epitope tag. GAPDH was used as control. Note the similar level of GlyRS ${ }^{\mathrm{CMT} 2 \mathrm{D}}$ and GlyRS ${ }^{\mathrm{WT}}$ in the media of transfected Cos7 cells. The observation that differentiated myotubes also secret GlyRS raises the possibility that muscles, which are directly innervated by the peripheral motor neurons, might contribute to the disease pathology.

a

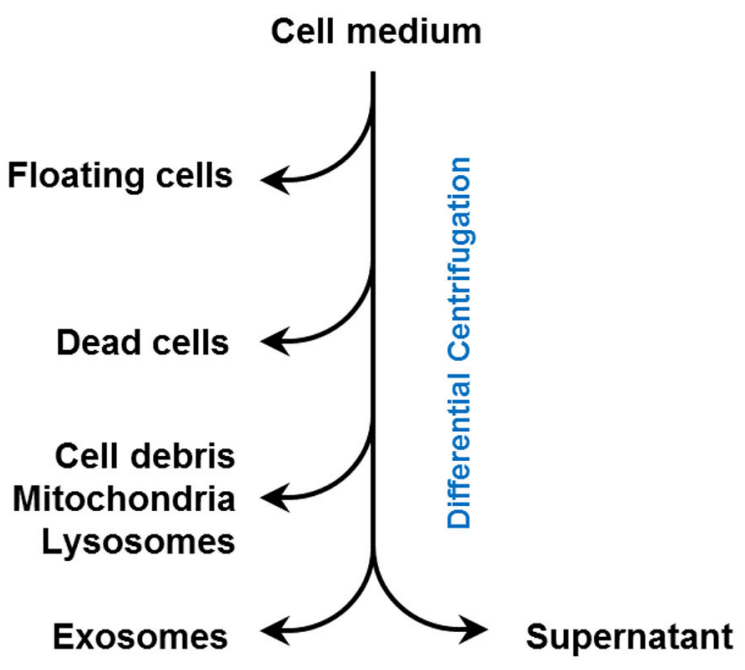

b

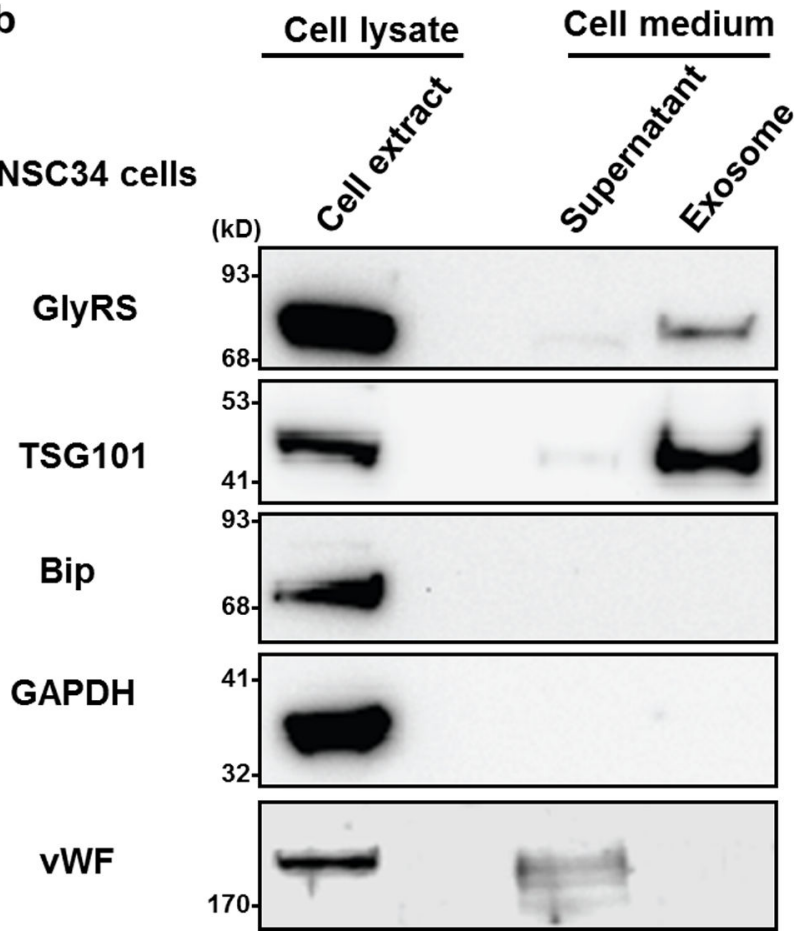

Extended Data Figure 4. Detection of GlyRS proteins in Exosome-enriched fractions

a, Diagram showing the procedure of "exosome" separation from the cell medium of NSC34 cells by differential centrifugation. See Materials and Methods for details. b, Western-blot analysis of proteins associated with various fractions. GlyRS proteins were detected in the "exosome"-enriched fractions but not in supernatant fractions. The quality of the "exosome" preparation was controlled by detection of TSG101 (exosomal protein), Bip (ER-associated protein), GAPDH (cytoplasmic protein), and vWF (secretory protein through ER-Golgi pathway). 
a
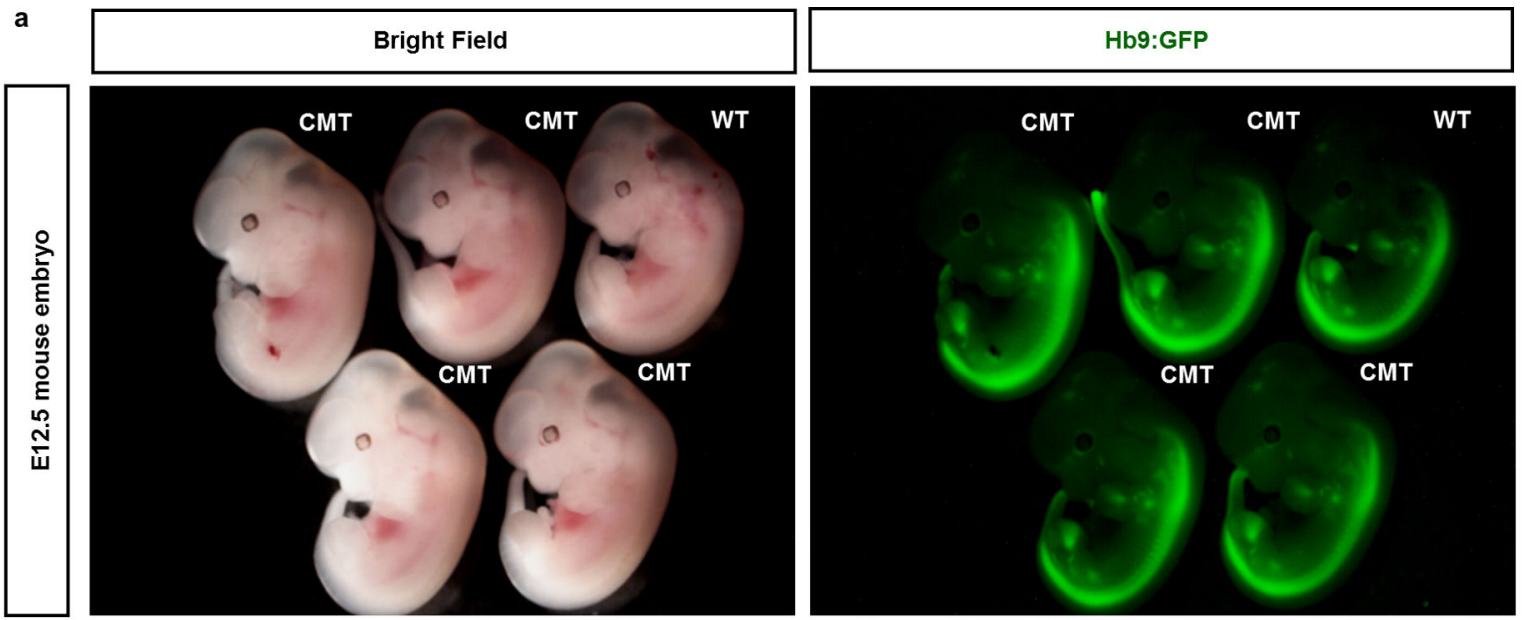

b
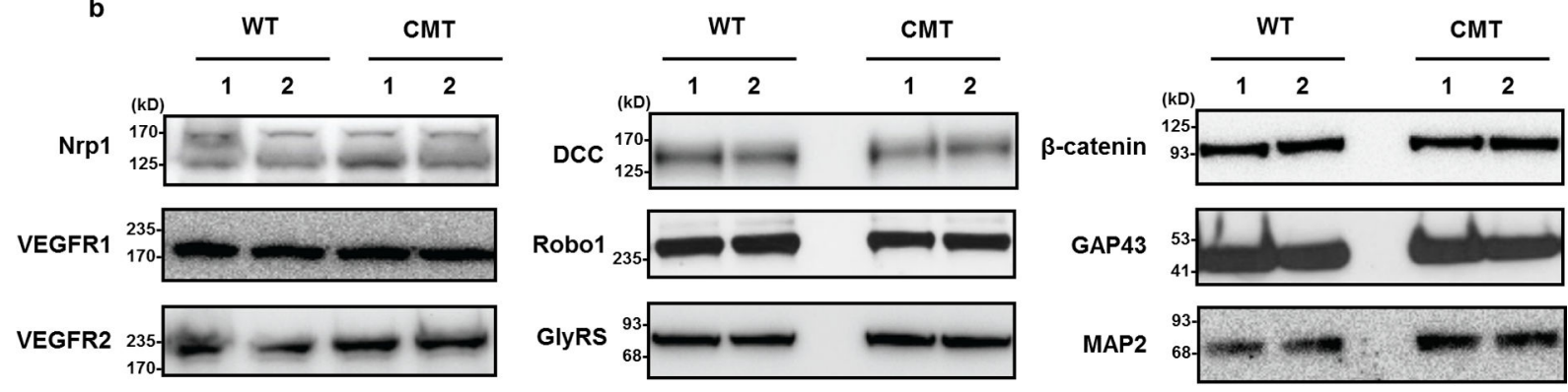

GAP43
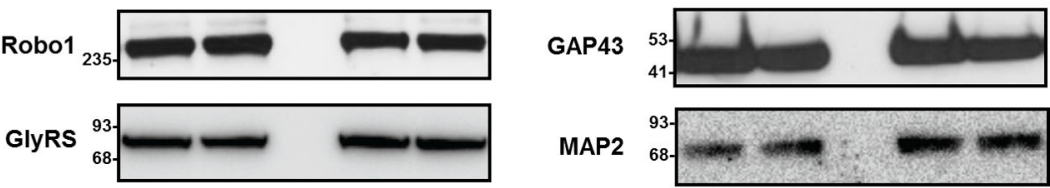

MAP2
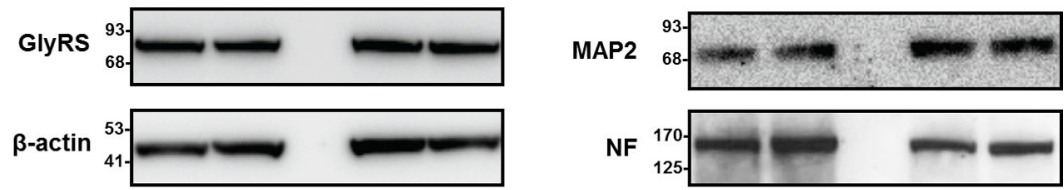

NF

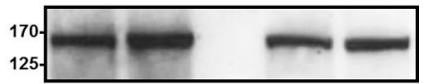

c

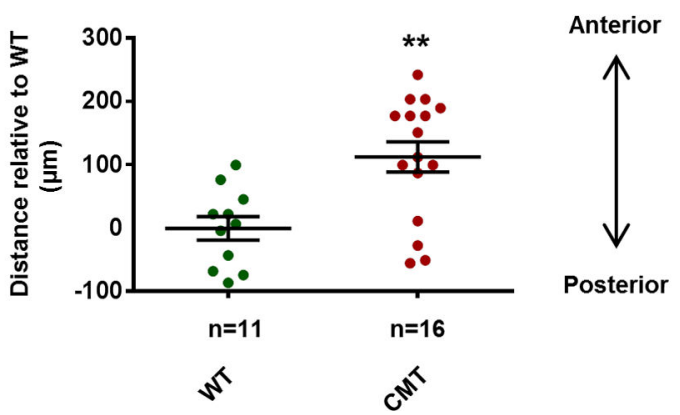

Extended Data Figure 5. CMT2D mutant embryos have overall normal morphology but exhibit facial motor neuron migration defects

a, Lateral view of WT and CMT2D mutant embryos at E12.5. Motor neurons are specifically labeled by a transgenic fluorescence reporter, Hb9:GFP (green). Note overall normal morphology of CMT2D mutant embryos (CMT) compared to their littermate controls (WT). b, Western-bolt analysis of protein expression in E12.5 mouse neural tissues. The expression levels of various neuronal proteins appear normal in CMT2D mutants compared to their littermate controls. c, The facial motor neuron migration phenotype is 
quantified by measuring the relative distance of the facial motor nucleus between WT and CMT littermate embryos (Each dot represents one facial motor nucleus, $n=6$ embryos for WT; $n=8$ embryos for CMT2D). We find the migration of facial motor neurons is significantly disrupted in CMT embryos. Data are presented as the mean \pm SEM. $* *$ p $<0.01$ (t-test).

a

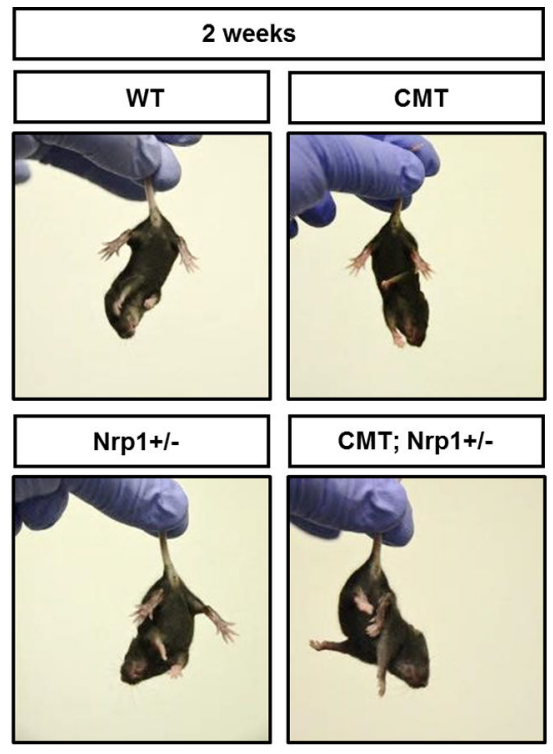

b

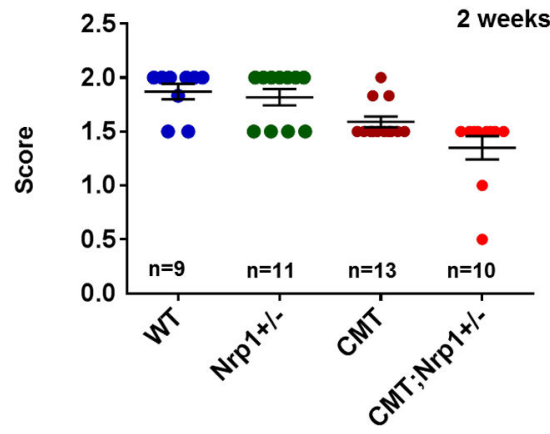

c

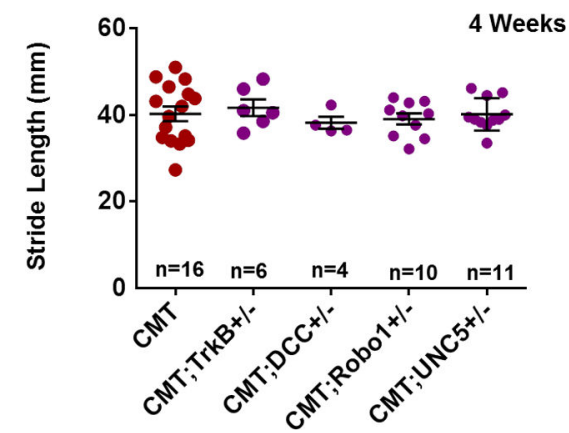

Extended Data Figure 6. Genetic-interaction between Gars and Nrp1 in the early stage of CMT2D 
a, b, Hindlimb extension test of wild-type and mutant animals at 2 weeks. Note that 2 out of

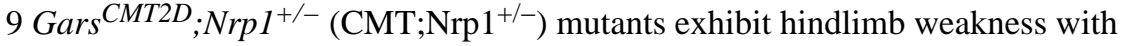
significantly lower scores compared to Gars ${ }^{C M T 2 D}$ (CMT), $\mathrm{Nrpl}^{+/-}\left(\mathrm{Nrp1}^{+/-}\right)$and wild-type (WT) littermate controls. $\mathbf{c}$, Comparison of stride lengths in different CMT2D mutant mice at 4-week-old: $\operatorname{Gars}^{C M T 2 D}$ (CMT), Gars $^{C M T 2 D}$;TrkB ${ }^{+/-}\left(\mathrm{CMT} \operatorname{TrkB}^{+/-}\right)$, Gars $^{\text {CMT2D }}$;DCC $C^{+/-}\left(\mathrm{CMT} ; \mathrm{DCC}^{+/-}\right)$, Gars $^{\text {CMT2D }^{2} \text { Robol }^{+/-}}\left(\mathrm{CMT} ;\right.$ Robol $\left.^{+/-}\right)$, and Gars $\left.^{\text {CMT2D }_{\text {Unc5 }} \mathrm{C}^{+/-}(\mathrm{CMT} \text {; Unc5C }} \mathrm{C}^{+/-}\right)$. No significant differences were observed between compound heterozygotes and their littermate controls (CMT). 

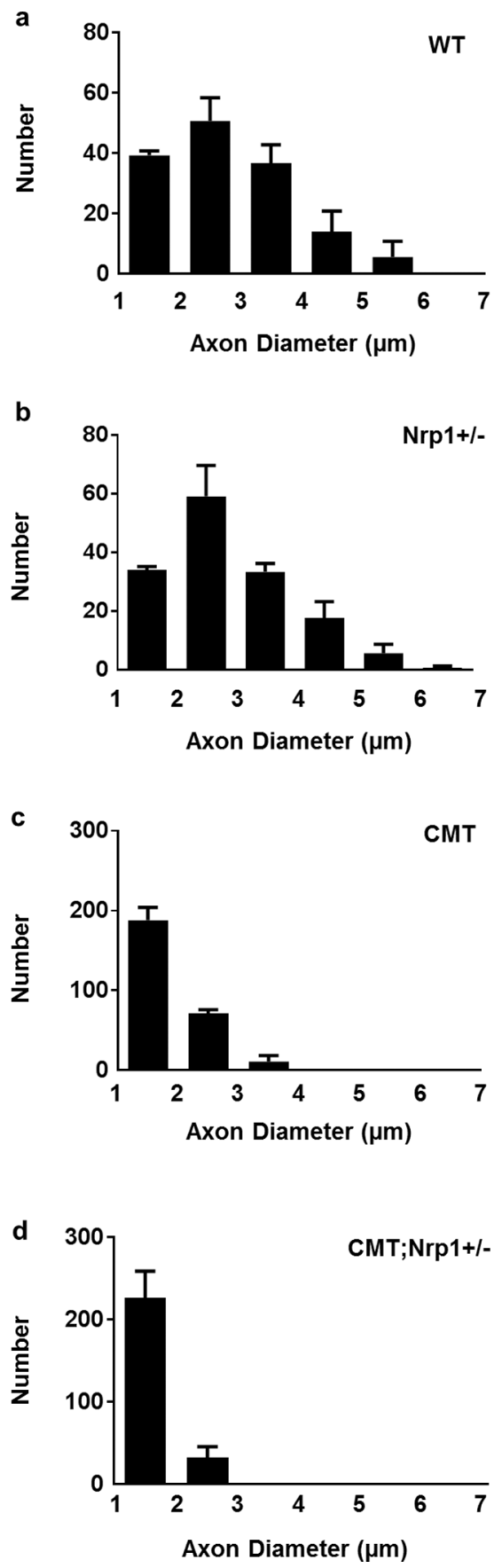

Extended Data Figure 7. Axonal dystrophy in CMT2D mice

Histogram showing the axonal diameter frequencies in the sciatic nerves of 4-week-old wild-type (WT, a), Nrpl heterozygous $\left(\mathrm{Nrp1}^{+/-}, \mathbf{b}\right)$, Gars $^{C M T 2 D}(\mathrm{CMT}, \mathbf{c})$, and (CMT; Nrp1 ${ }^{+/-}$, d) mutant mice. $\mathrm{n}=3$ mice per group. Note the decreased numbers of largerdiameter axons in CMT; Nrp1 $1^{+/-}$mutants compared to CMT, Nrp1 heterozygous, and wildtype controls. 

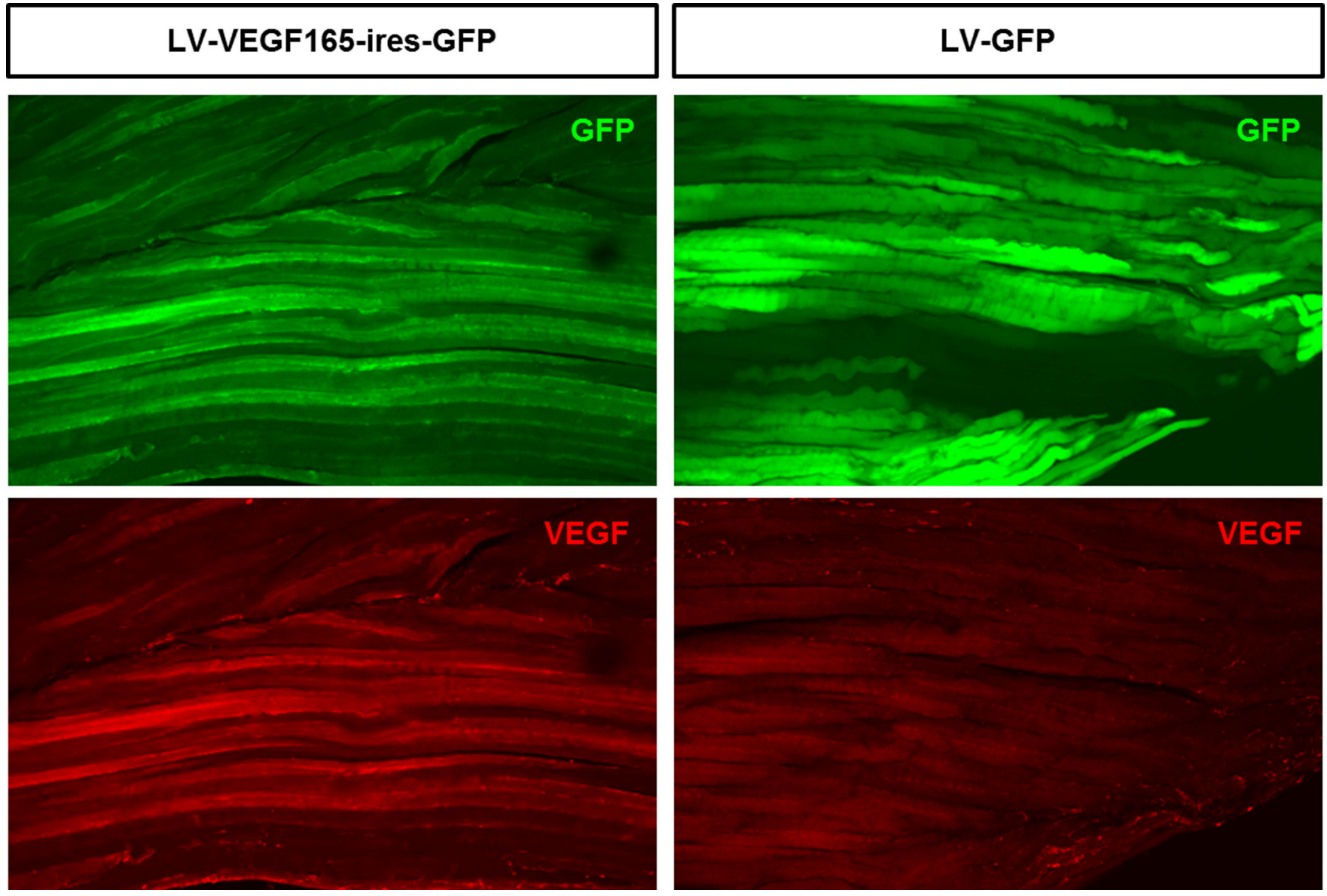

Extended Data Figure 8. Expression level of VEGF in mouse muscles

The expression level of VEGF proteins in muscle fibers of mice injected with lentivirus expressing LV-VEGF165-ires-GFP versus LV-GFP was determined by immunostaining with anti-VEGF antibodies. Note the expression level of VEGF in LV-VEGF infected muscles is significantly higher than in LV-GFP infected control groups. 
a

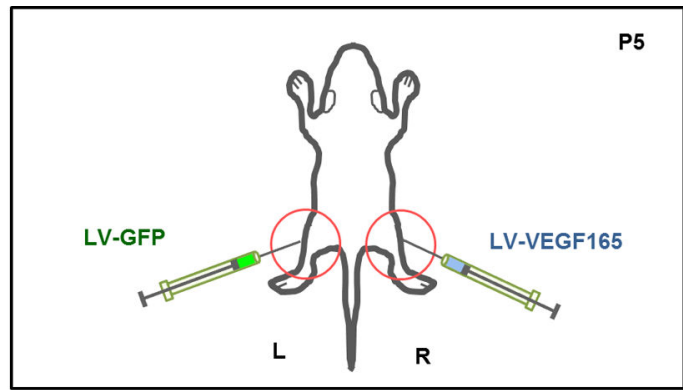

b

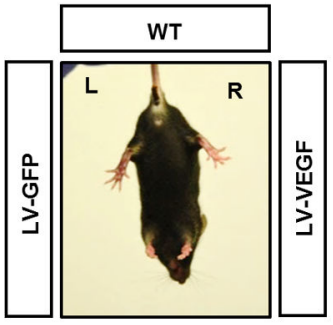

d

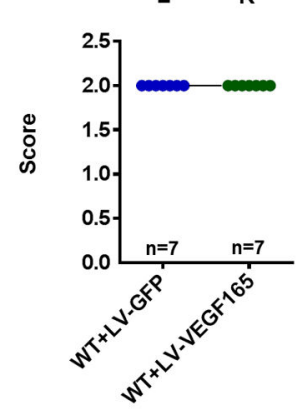

f

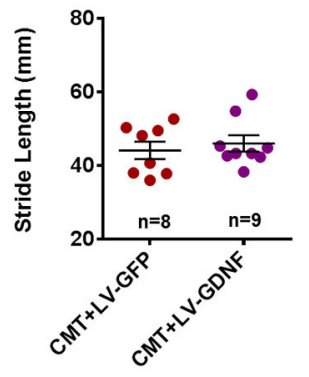

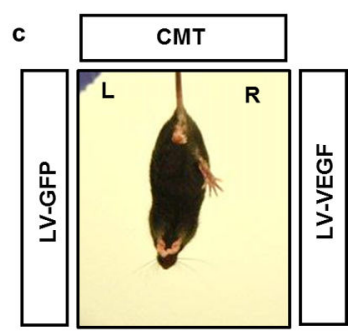

e

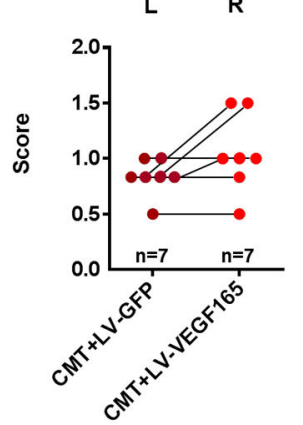

g

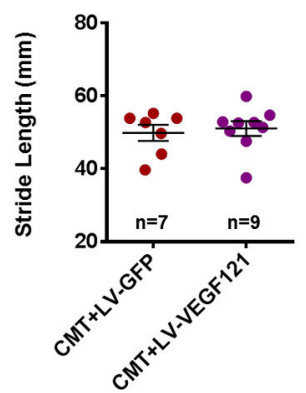

Extended Data Figure 9. VEGF treatment retains limb strength in CMT2D mice a, Diagram showing that lentiviral vectors encoding GFP (LV-GFP) or VEGF-A165 (LVVEGF165) are injected unilaterally into each hindlimb of the same GlyRS $C M T 2 D$ mutant mouse at P5. c, e, At 5 weeks, LV-GFP-injected legs (L, left) of CMT2D animals have largely lost their ability to extend, while LV-VEGF165-treated legs (R, right) retained more limb strength with significantly higher scores in the hindlimb extension test (3 out of 7 animals). $\mathrm{p}<0.05$ (Permutation test). No significant difference was observed between both injected legs of wild-type animals in the hindlimb extension test (b, d). f, g, GDNF and 
VEGF- $\mathrm{A}_{121}$ treatments fail to improve stride length in CMT2D mice. Walking strides of 2month-old CMT2D mice bilaterally injected with lentiviral vectors (LV) encoding GFP, GDNF or VEGF-A $A_{121}$. No significant difference of hindlimb stride length was observed between animals treated with LV-GDNF, LV-VEGF-A 121 , and LV-GFP controls.

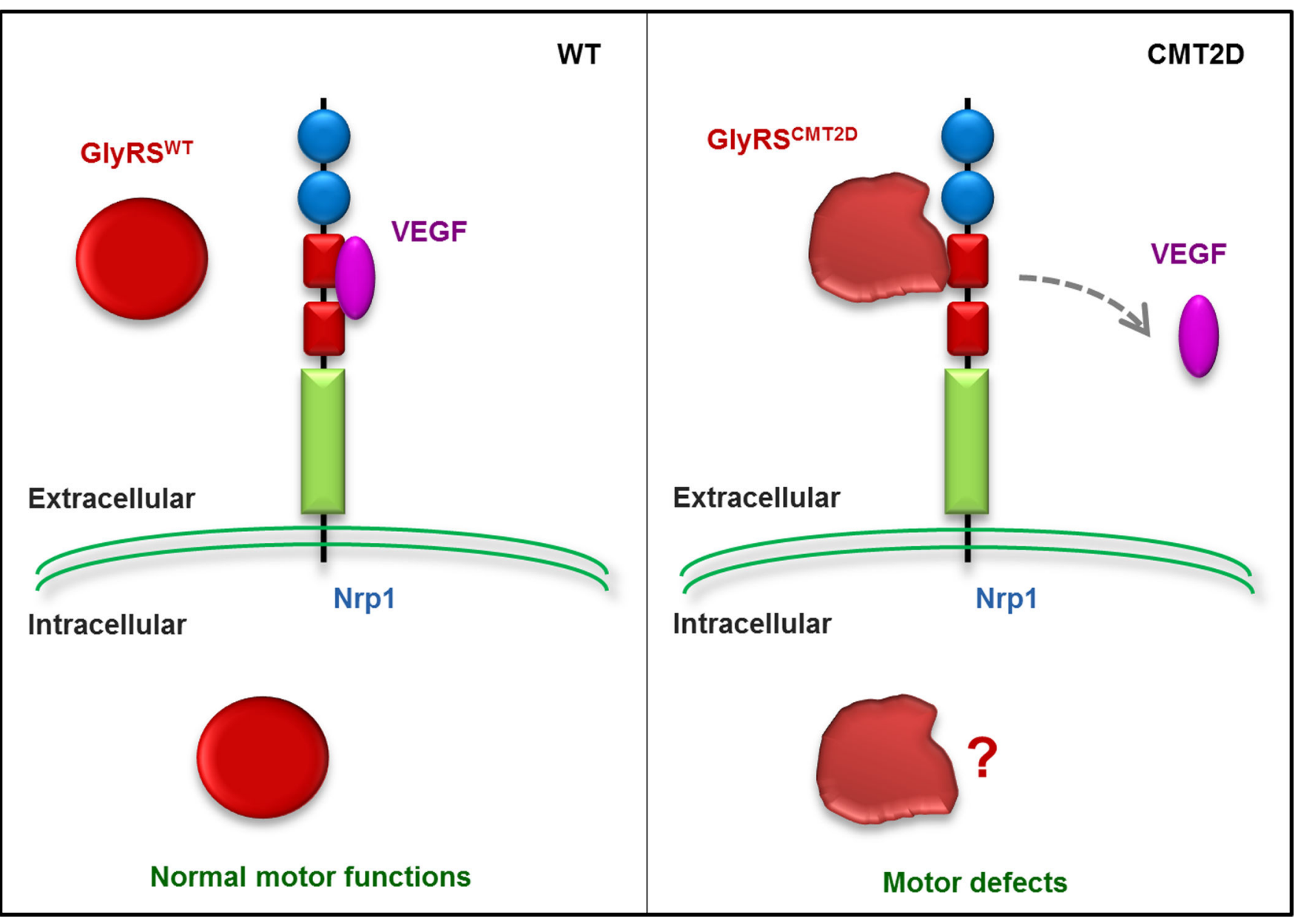

Extended Data Figure 10. A simplified model for the neomorphic binding activity of GlyRS CMT2D

Left panel, GlyRS ${ }^{\mathrm{WT}}$ is a multifunctional protein with both intracellular and extracellular distributions. VEGF/Nrp1 signaling is an essential pathway for survival and function of motor neurons. (Note that VEGF may also act synergistically with other trophic factors, and/or maintains motor-function indirectly by acting on Nrp1 receptors on non-motor neurons.) Right panel, CMT2D mutations alter the conformation of GlyRS, enabling GlyRS CMT2D to bind Nrp1. This aberrant interaction antagonizes the binding of VEGF to Nrp1, contributing to motor defects in CMT2D. Our results do not exclude the possibility that GlyRSCMT2D may also interact with other extracellular and/or intracellular targets, related to CMT2D pathology.

\section{Supplementary Material}

Refer to Web version on PubMed Central for supplementary material. 


\section{Acknowledgments}

We thank P. Schimmel, J. Dolkas, C. Farrokhi, L. Lisowski, C. Ly, S. Chalasani, T. Liu, Q. Hu, P. Li, N. Sheng and members of The Scripps Laboratories of tRNA Synthetase Research and the Pfaff laboratory for advice and discussions on the experiments and manuscript. We are grateful to S. Ackerman, K-F. Lee, D. O'leary and D. Ginty for providing mouse lines. W.H., H.Z. and N.W. were supported by fellowships from National Foundation for Cancer Research. G.B was supported by the Pioneer fund and Howard Hughes Medical Institute. S.L.P. is a Howard Hughes Medical Institute investigator and a Benjamin H. Lewis chair in Neuroscience. This research is supported by grants from the US National Institutes of Health (R01GM088278, R21NS084254, and R01NS054154), The Marshall Heritage Foundation, and Sol Goldman Trust, and by aTyr Pharma through an agreement with The Scripps Research Institute.

X.-L.Y. is a scientific cofounder and consultant of aTyr Pharma.

\section{References}

1. Saxena S, Caroni P. Selective neuronal vulnerability in neurodegenerative diseases: from stressor thresholds to degeneration. Neuron. 2011; 71:35-48. [PubMed: 21745636]

2. Skre H. Genetic and clinical aspects of Charcot-Marie-Tooth's disease. Clin Genet. 1974; 6:98-118. [PubMed: 4430158]

3. Patzko A, Shy ME. Update on Charcot-Marie-Tooth disease. Curr Neurol Neurosci Rep. 2011; 11:78-88. [PubMed: 21080241]

4. Antonellis A, et al. Glycyl tRNA synthetase mutations in Charcot-Marie-Tooth disease type 2D and distal spinal muscular atrophy type V. Am J Hum Genet. 2003; 72:1293-1299. [PubMed: 12690580]

5. Wakasugi K, Schimmel P. Two distinct cytokines released from a human aminoacyl-tRNA synthetase. Science. 1999; 284:147-151. [PubMed: 10102815]

6. Yao P, Fox PL. Aminoacyl-tRNA synthetases in medicine and disease. EMBO Mol Med. 2013; 5:332-343. [PubMed: 23427196]

7. Williams TF, Mirando AC, Wilkinson B, Francklyn CS, Lounsbury KM. Secreted Threonyl-tRNA synthetase stimulates endothelial cell migration and angiogenesis. Sci Rep. 2013; 3:1317. [PubMed: 23425968]

8. Park MC, et al. Secreted human glycyl-tRNA synthetase implicated in defense against ERKactivated tumorigenesis. Proc Natl Acad Sci U S A. 2012; 109:E640-E647. [PubMed: 22345558]

9. Guo M, Yang XL, Schimmel P. New functions of aminoacyl-tRNA synthetases beyond translation. Nat Rev Mol Cell Biol. 2010; 11:668-674. [PubMed: 20700144]

10. Lee HJ, et al. Two novel mutations of GARS in Korean families with distal hereditary motor neuropathy type V. J Peripher Nerv Syst. 2012; 17:418-421. [PubMed: 23279345]

11. Motley WW, Talbot K, Fischbeck KH. GARS axonopathy: not every neuron's cup of tRNA. Trends Neurosci. 2010; 33:59-66. [PubMed: 20152552]

12. Kawakami N, et al. A novel mutation in glycyl-tRNA synthetase caused Charcot-Marie-Tooth disease type 2D with facial and respiratory muscle involvement. Rinsho Shinkeigaku. 2014; 54:911-915. [PubMed: 25420567]

13. Sun A, et al. A novel mutation of the glycyl-tRNA synthetase (GARS) gene associated with Charcot-Marie-Tooth type 2D in a Chinese family. Neurol Res. 2015; 37:782-787. [PubMed: 26000875]

14. Achilli F, et al. An ENU-induced mutation in mouse glycyl-tRNA synthetase (GARS) causes peripheral sensory and motor phenotypes creating a model of Charcot-Marie-Tooth type 2D peripheral neuropathy. Dis Model Mech. 2009; 2:359-373. [PubMed: 19470612]

15. Seburn KL, Nangle LA, Cox GA, Schimmel P, Burgess RW. An active dominant mutation of glycyl-tRNA synthetase causes neuropathy in a Charcot-Marie-Tooth 2D mouse model. Neuron. 2006; 51:715-726. [PubMed: 16982418]

16. Motley WW, et al. Charcot-Marie-Tooth-linked mutant GARS is toxic to peripheral neurons independent of wild-type GARS levels. PLoS Genet. 2011; 7:e1002399. [PubMed: 22144914] 
17. He W, et al. Dispersed disease-causing neomorphic mutations on a single protein promote the same localized conformational opening. Proc Natl Acad Sci U S A. 2011; 108:12307-12312. [PubMed: 21737751]

18. Del Bo R, et al. Coexistence of CMT-2D and distal SMA-V phenotypes in an Italian family with a GARS gene mutation. Neurology. 2006; 66:752-754. [PubMed: 16534118]

19. Dubourg O, et al. The G526R glycyl-tRNA synthetase gene mutation in distal hereditary motor neuropathy type V. Neurology. 2006; 66:1721-1726. [PubMed: 16769947]

20. Schwarz Q, et al. Vascular endothelial growth factor controls neuronal migration and cooperates with Sema3A to pattern distinct compartments of the facial nerve. Genes Dev. 2004; 18:28222834. [PubMed: 15545635]

21. Huber AB, et al. Distinct roles for secreted semaphorin signaling in spinal motor axon guidance. Neuron. 2005; 48:949-964. [PubMed: 16364899]

22. Song MR, et al. T-Box transcription factor Tbx20 regulates a genetic program for cranial motor neuron cell body migration. Development. 2006; 133:4945-4955. [PubMed: 17119020]

23. Lewcock JW, Genoud N, Lettieri K, Pfaff SL. The ubiquitin ligase Phr1 regulates axon outgrowth through modulation of microtubule dynamics. Neuron. 2007; 56:604-620. [PubMed: 18031680]

24. Mackenzie F, Ruhrberg C. Diverse roles for VEGF-A in the nervous system. Development. 2012; 139:1371-1380. [PubMed: 22434866]

25. Oosthuyse B, et al. Deletion of the hypoxia-response element in the vascular endothelial growth factor promoter causes motor neuron degeneration. Nat Genet. 2001; 28:131-138. [PubMed: 11381259]

26. Wang LJ, et al. Neuroprotective effects of glial cell line-derived neurotrophic factor mediated by an adeno-associated virus vector in a transgenic animal model of amyotrophic lateral sclerosis. $\mathbf{J}$ Neurosci. 2002; 22:6920-6928. [PubMed: 12177190]

27. Acsadi G, et al. Increased survival and function of SOD1 mice after glial cell-derived neurotrophic factor gene therapy. Hum Gene Ther. 2002; 13:1047-1059. [PubMed: 12067438]

28. Kaspar BK, Llado J, Sherkat N, Rothstein JD, Gage FH. Retrograde viral delivery of IGF-1 prolongs survival in a mouse ALS model. Science. 2003; 301:839-842. [PubMed: 12907804]

29. Nayak MS, Kim YS, Goldman M, Keirstead HS, Kerr DA. Cellular therapies in motor neuron diseases. Biochim Biophys Acta. 2006; 1762:1128-1138. [PubMed: 16872810]

30. Azzouz M, et al. VEGF delivery with retrogradely transported lentivector prolongs survival in a mouse ALS model. Nature. 2004; 429:413-417. [PubMed: 15164063]

\section{References}

31. Lee SK, Jurata LW, Funahashi J, Ruiz EC, Pfaff SL. Analysis of embryonic motoneuron gene regulation: derepression of general activators function in concert with enhancer factors. Development. 2004; 131:3295-3306. [PubMed: 15201216]

32. Gu C, et al. Neuropilin-1 conveys semaphorin and VEGF signaling during neural and cardiovascular development. Dev Cell. 2003; 5:45-57. [PubMed: 12852851]

33. $\mathrm{Xu} \mathrm{B}$, et al. Cortical degeneration in the absence of neurotrophin signaling: dendritic retraction and neuronal loss after removal of the receptor TrkB. Neuron. 2000; 26:233-245. [PubMed: 10798407]

34. Ma L, Tessier-Lavigne M. Dual branch-promoting and branch-repelling actions of Slit/Robo signaling on peripheral and central branches of developing sensory axons. J Neurosci. 2007; 27:6843-6851. [PubMed: 17581972]

35. Fazeli A, et al. Phenotype of mice lacking functional Deleted in colorectal cancer (Dcc) gene. Nature. 1997; 386:796-804. [PubMed: 9126737]

36. Burgess RW, Jucius TJ, Ackerman SL. Motor axon guidance of the mammalian trochlear and phrenic nerves: dependence on the netrin receptor Unc5c and modifier loci. J Neurosci. 2006; 26:5756-5766. [PubMed: 16723533]

37. Chalmers MJ, et al. Probing protein ligand interactions by automated hydrogen/deuterium exchange mass spectrometry. Anal Chem. 2006; 78:1005-1014. [PubMed: 16478090] 
38. Pascal BD, et al. HDX workbench: software for the analysis of H/D exchange MS data. J Am Soc Mass Spectrom. 2012; 23:1512-1521. [PubMed: 22692830]

39. Ericson J, Thor S, Edlund T, Jessell TM, Yamada T. Early stages of motor neuron differentiation revealed by expression of homeobox gene Islet-1. Science. 1992; 256:1555-1560. [PubMed: 1350865]

40. Savina A, Furlan M, Vidal M, Colombo MI. Exosome release is regulated by a calcium-dependent mechanism in K562 cells. J Biol Chem. 2003; 278:20083-20090. [PubMed: 12639953]

41. Soo CY, et al. Nanoparticle tracking analysis monitors microvesicle and exosome secretion from immune cells. Immunology. 2012; 136:192-197. [PubMed: 22348503] 
a

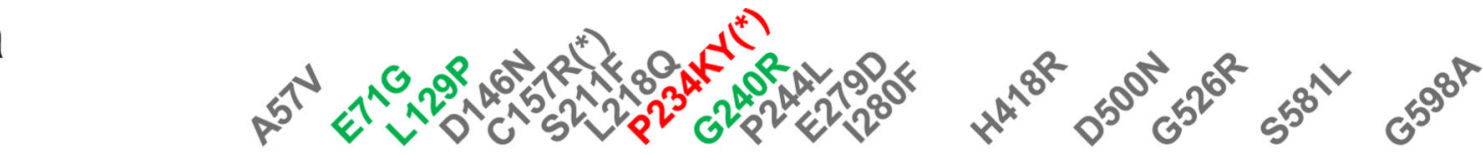

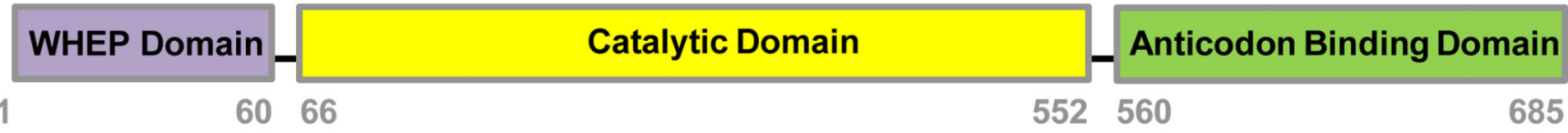

b GlyRS $^{\text {CMT2D }}$ in patients

C P234KY-GlyRS ${ }^{\mathrm{CMT2D}}$ in mice

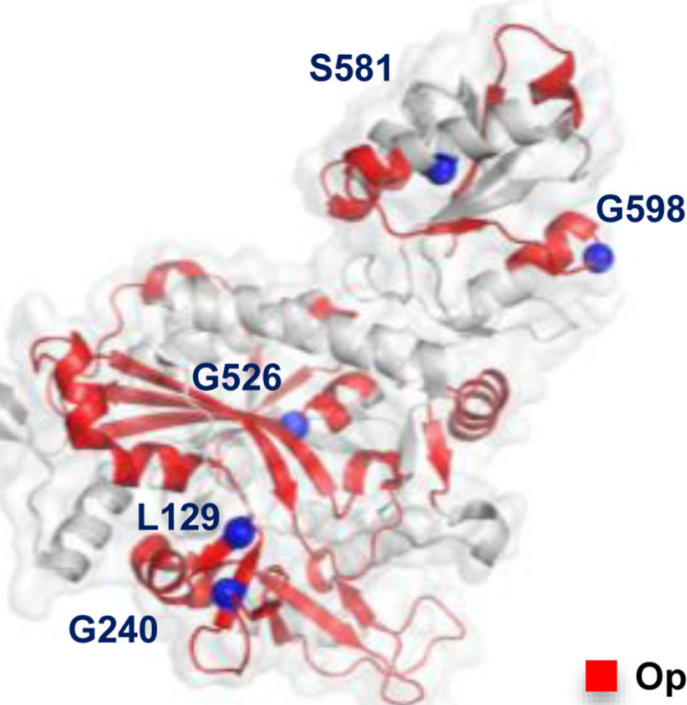

Opened surface

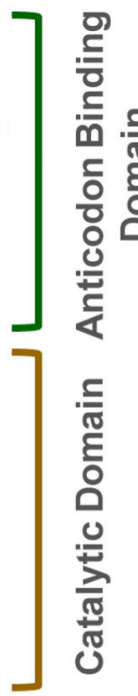

- CMT2D-associated residue

Figure 1. Dispersed CMT2D mutations consistently cause neomorphic structural opening at the dimer interface of GlyRS

a, Distribution of 15 CMT2D-associated dominant mutations in the three domains of the cytosolic human GlyRS. The 3 strongest pathogenic mutations are highlighted in green. Two mutations identified in mice $\left(^{*}\right)$ are labeled with their corresponding residue numbers in the human protein. b, Human GlyRS structure (monomeric subunit) viewed from dimer interface. Consensus opened-up areas caused by 5 CMT2D mutations are labeled in red ${ }^{17}$. c, Opened-up areas (red) by the P234KY mutation (>10 \% increase in deuterium incorporation relative to WT GlyRS.) 


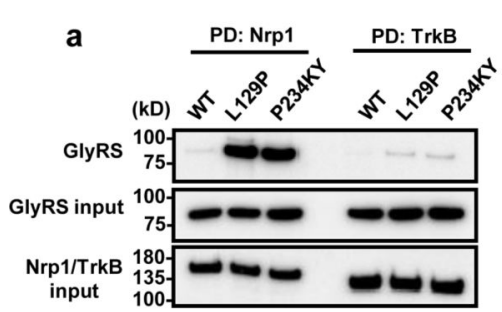

b Tissue lysate IP: IgG anti-Nrp1
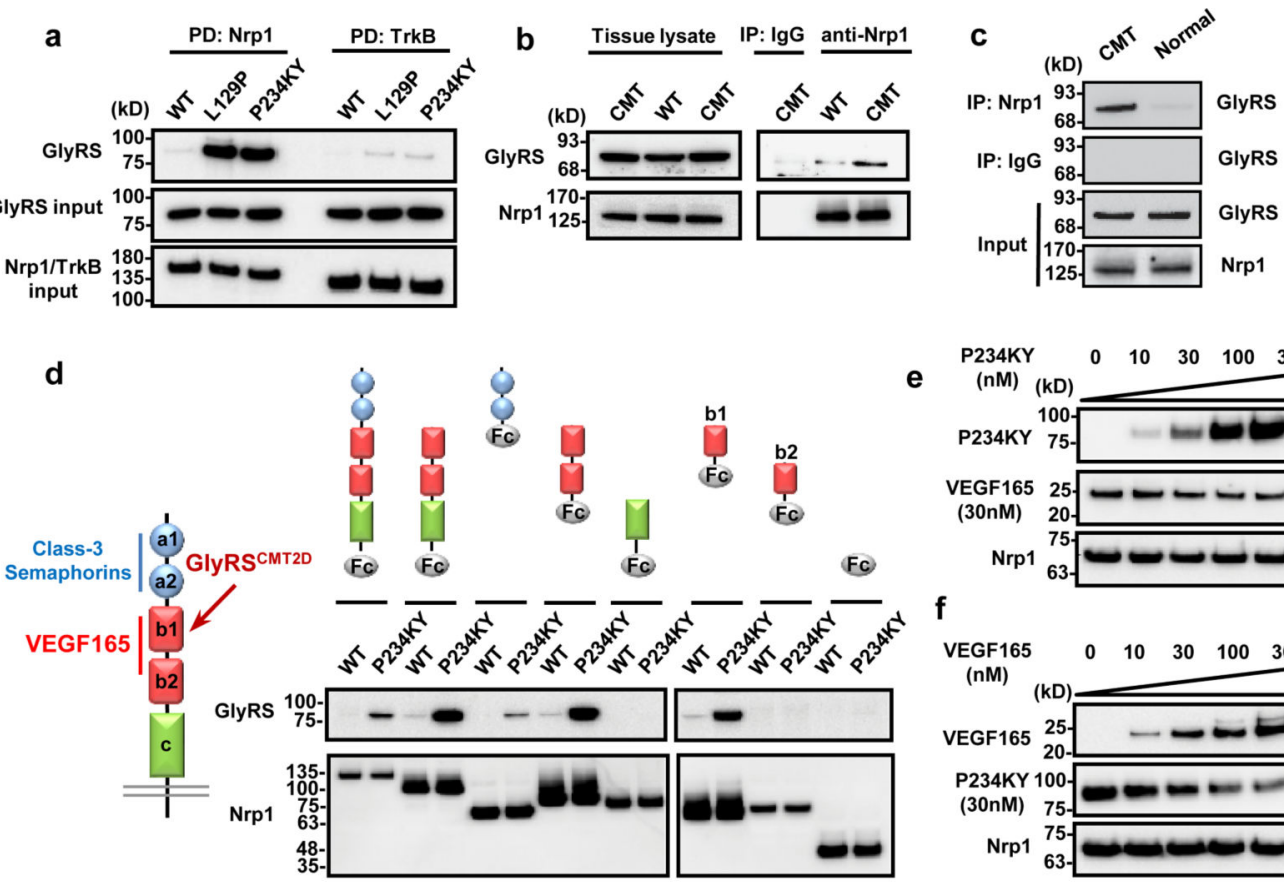

$\begin{array}{lllllll}\text { P234KY } & 0 & 10 & 30 & 100 & 300\end{array}$
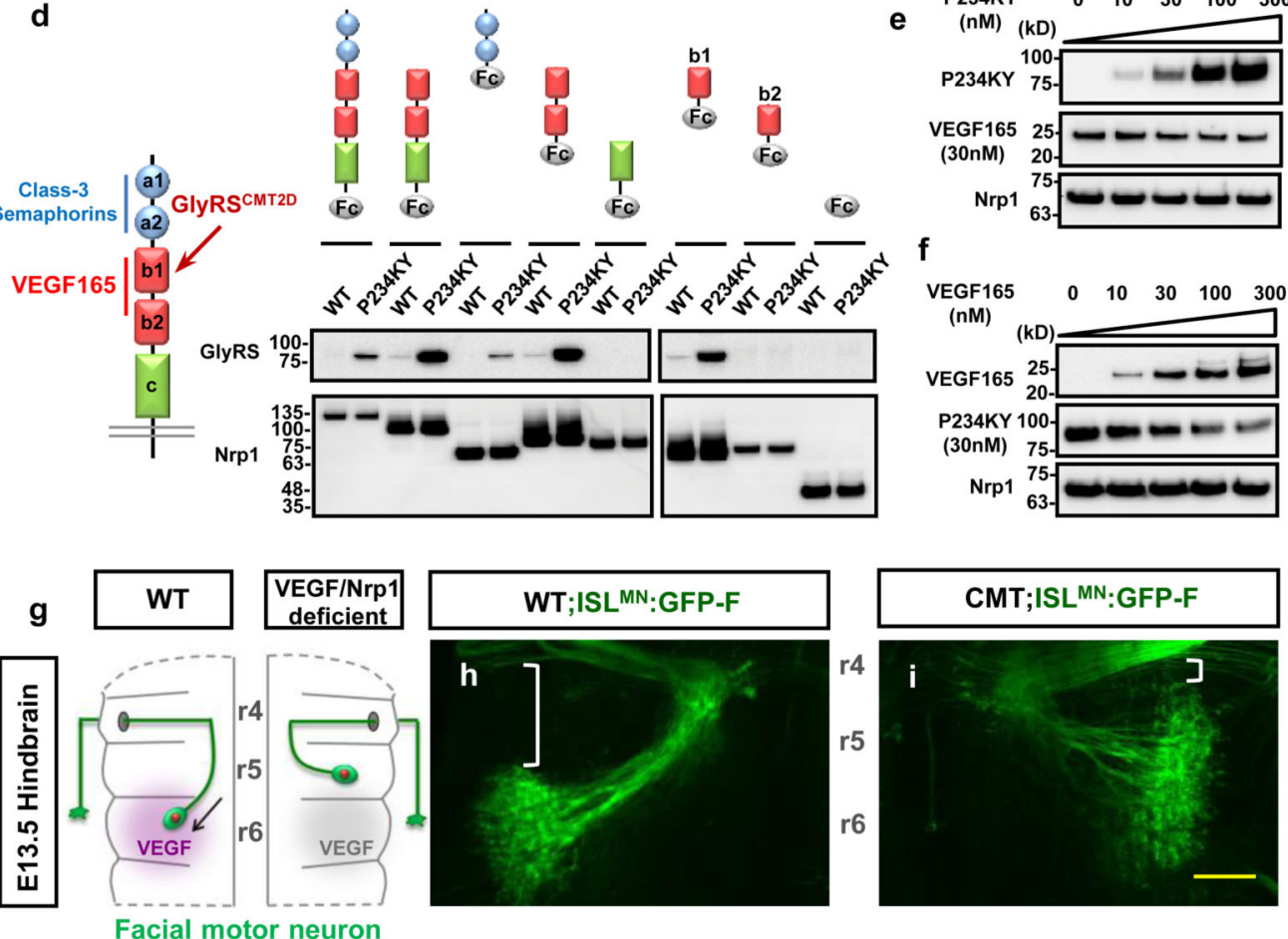

Facial motor neuron
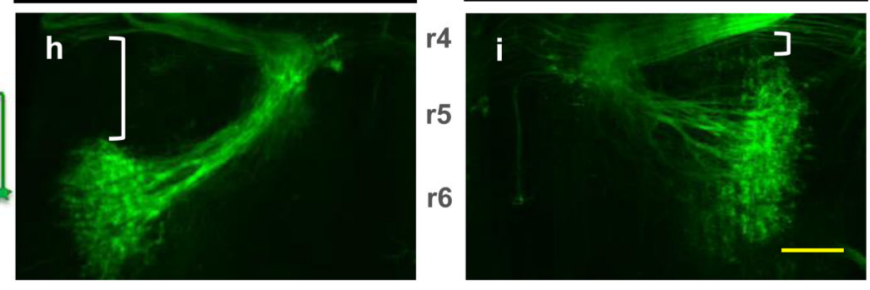

migration
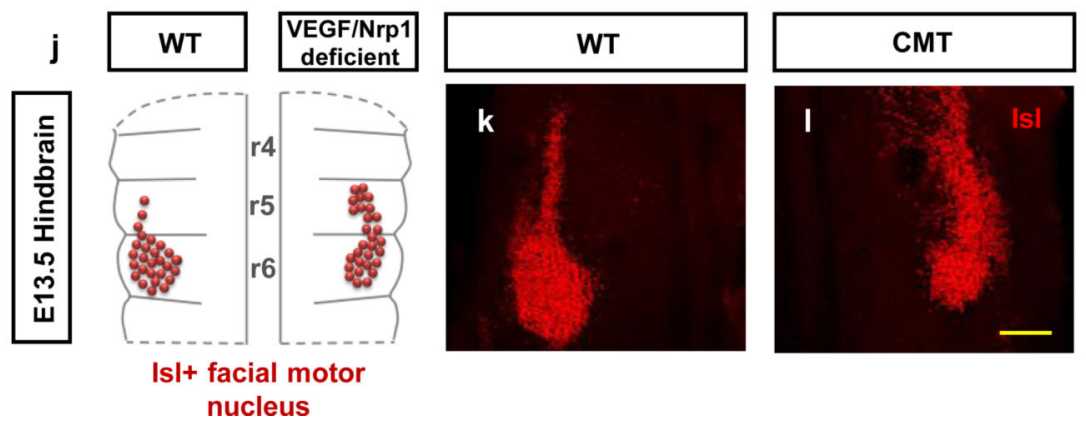

Figure 2. GlyRS CMT2D specifically binds Nrp1 and antagonizes VEGF-Nrp1 interaction a, In vitro pull-down (PD) of GlyRS ${ }^{\text {CMT2D }}$ proteins by the ectodomain of Nrp1 but not TrkB. b, Co-immunoprecipitation (IP) to detect GlyRS-Nrp1 interaction in neural tissues of wild-type (WT) and P234KY-Gars ${ }^{C M T 2 D}$ mice (CMT). c, Co-immunoprecipitation (IP) to detect GlyRS-Nrp1 interaction in lymphocytes from CMT2D patients carrying the L129P mutation $(n=5)$ and from healthy individuals $(n=3)$. $\mathbf{d}$, Domain mapping using in vitro IP identifies the b1 domain of Nrp1 as the main binding site of GlyRS ${ }^{\text {CMT2D }}$. e, $\mathbf{f}$, In vitro PD assay showing the competition between $\mathrm{P} 234 \mathrm{KY}-\mathrm{GlyRS} \mathrm{CMT}^{2 \mathrm{D}}$ and VEGF-A 165 proteins for 
Nrp1 (b domains) binding. $\mathbf{g}, \mathbf{j}$, Schematic of facial motor neuron migration (g) and facial motor nucleus $(\mathbf{j})$ in open-book preparations of WT (left half) and VEGF/Nrp1-deficient mouse hindbrains at E13.5 (right half) ${ }^{20} . \mathbf{h}, \mathbf{i}$, Fluorescence labeling of facial motor neuron somata and axons by ISL ${ }^{\mathrm{MN}}$ :GFP-F on one side of E13.5 mouse hindbrain of open-book preparation. $\mathbf{k}, \mathbf{l}$, Immunostaining of Isl-positive facial nucleus on one side of the E13.5 mouse hindbrain of open-book preparation. Scale bar represents $200 \mu \mathrm{m}$ in $\mathbf{h}, \mathbf{i}, \mathbf{k}, \mathbf{l}$. 
a

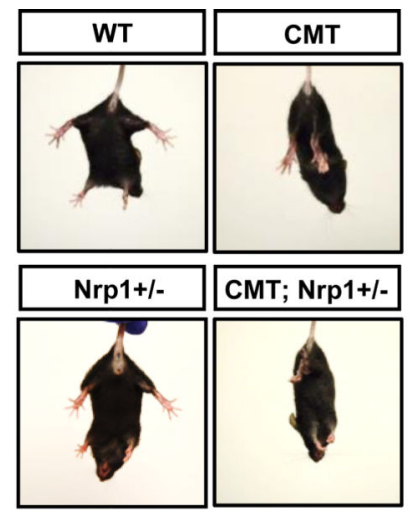

C

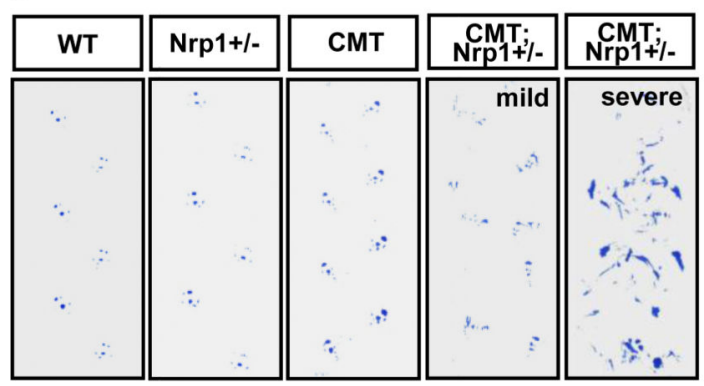

b

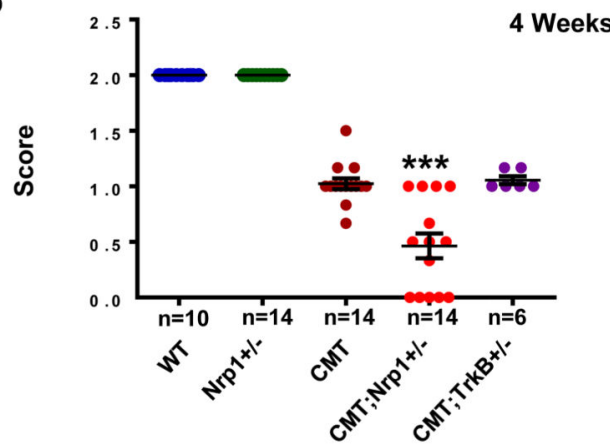

e
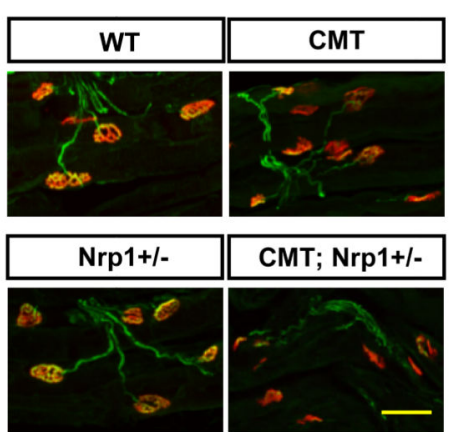

g

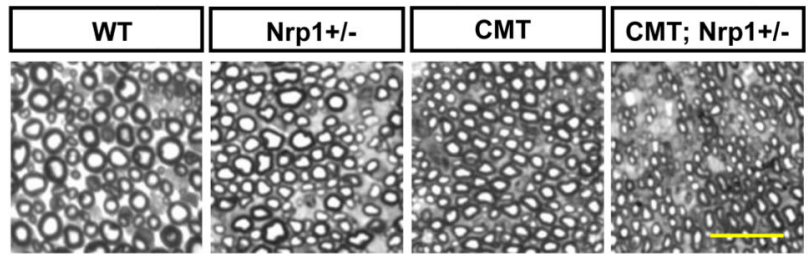

d
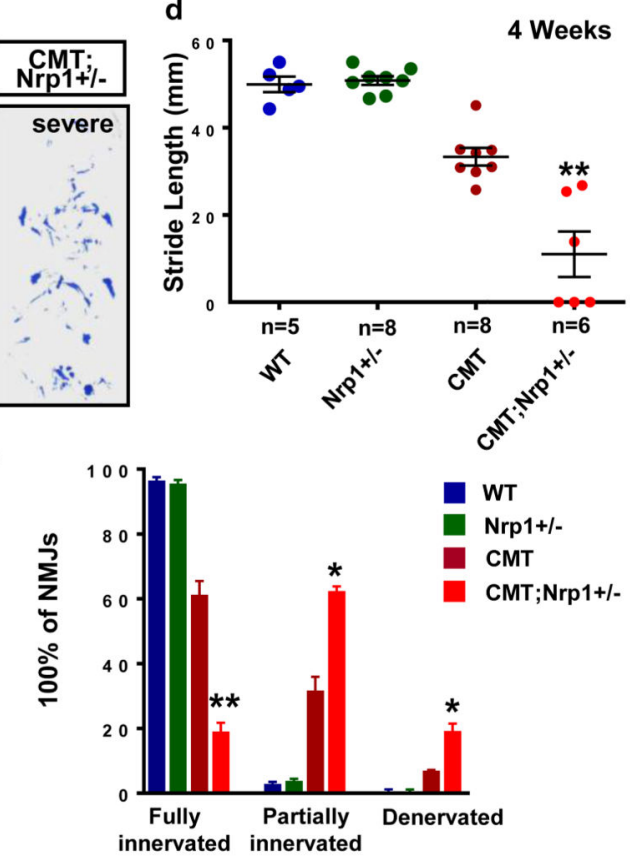

h $\mathbf{W T} \quad \mathbf{C M T}$

$\mathrm{h}$ Nrp1+l- $\mathrm{CMT} ; \mathrm{Nrp1+l-}$

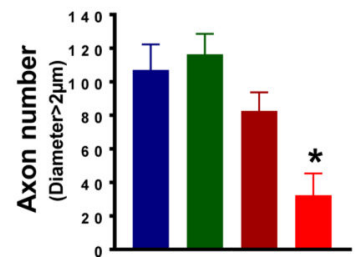

Figure 3. Nrp1 is a genetic modifier of CMT2D

a, b, Hindlimb extension test at 4 weeks. $* * *$ p $<0.001$ (Mann-Whitney test). c, Hindlimb footprints of WT and mutant animals at 4 weeks. Gars $\mathrm{CMT}^{\mathrm{D}} / \mathrm{Nrpl}^{+/-}$mutant mice exhibit disrupted gait patterns of different degrees (mild, severe). Note that severe cases show inability to walk. d, Stride length of WT and mutant animals at 4 weeks. e, f, Neuromuscular junction (NMJ) immunostaining in the gastrocnemius muscles of 4-week-old mice with the motor nerve terminal labeled in green and acetylcholine receptors on the muscle labeled in red. Data are presented as mean values \pm SEM. $n=3$ mice per group. Scale bar represents 
$50 \mu \mathrm{m} . \mathbf{g}, \mathbf{h}$, Myelinated axons from sciatic nerves of 4-week-old mice. Scale bar represents $20 \mu \mathrm{m}$. Histogram showing the quantification of axon numbers with the diameter larger than $2 \mu \mathrm{m}(\mathbf{h}) . \mathrm{n}=3$ mice per group. $* \mathrm{p}<0.05, * * \mathrm{p}<0.01$ (t-test) for $\mathbf{d}, \mathbf{f}$ and $\mathbf{h}$. 

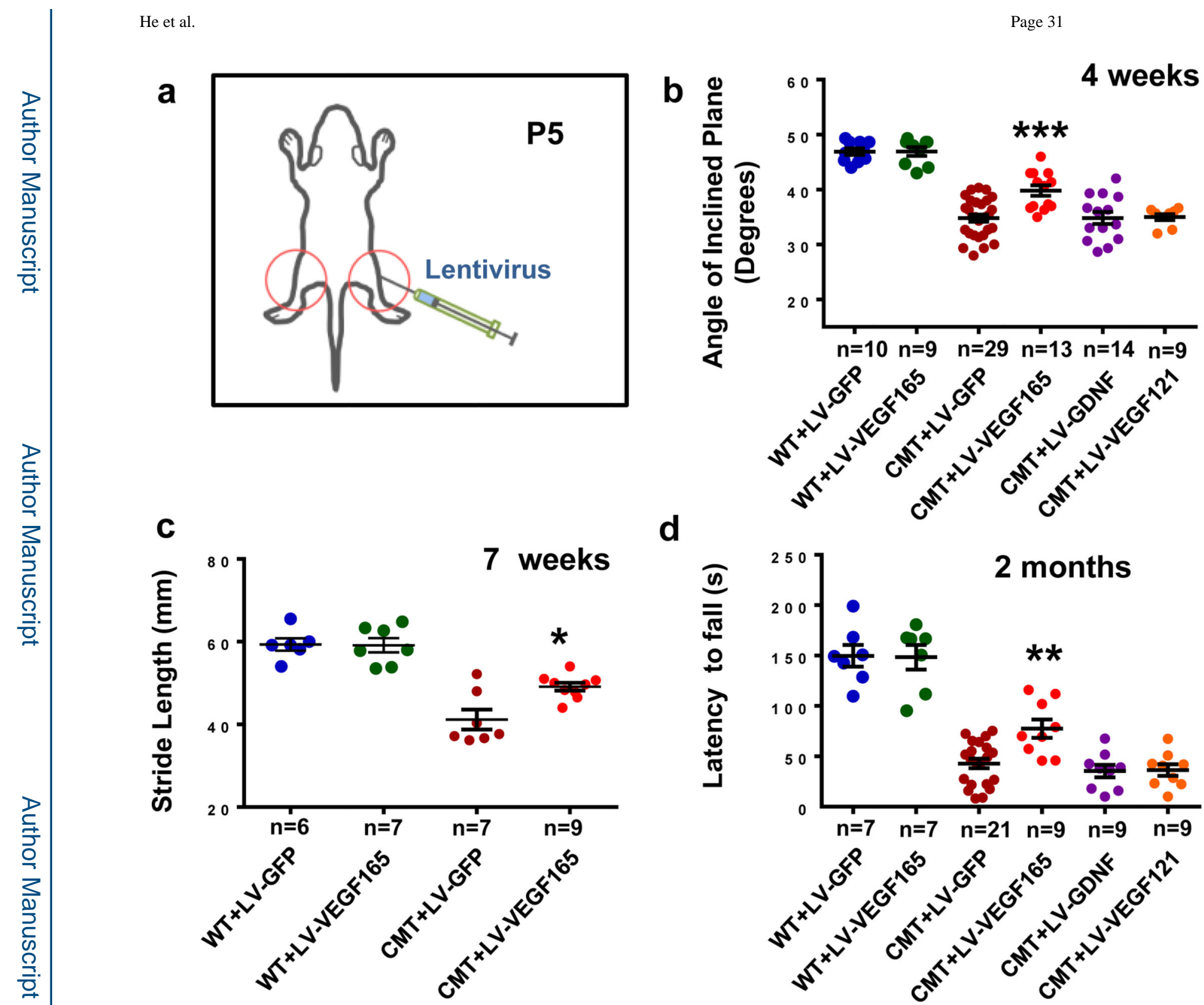

d

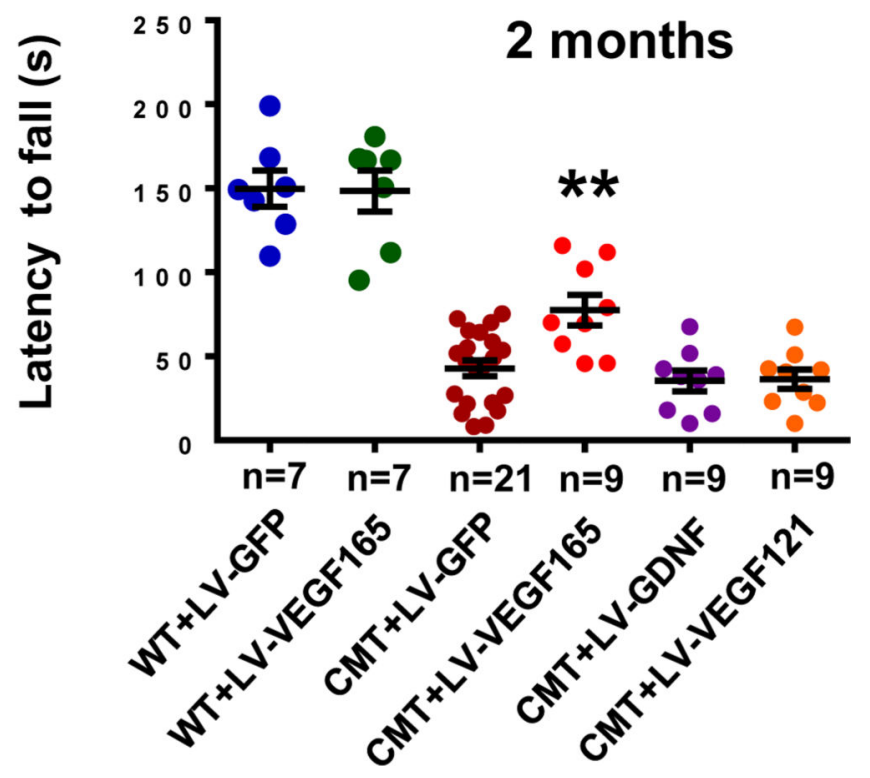

Figure 4. VEGF treatment improves motor function in CMT2D mice

a, Diagram showing bilateral intramuscular injection of lentivirus (LV) into mouse hindlimbs at P5. b, Inclined plane test of 4-week-old animals. c, Walking strides of 7-weekold animals. d, Rotarod test of 2 -month-old animals. ${ }^{*} \mathrm{p}<0.05$, **p $<0.01$, ***p $<0.001(\mathrm{t}$ test) for $\mathbf{b}, \mathbf{c}$ and $\mathbf{d}$. 